\title{
Real-time coupled-cluster approach for the cumulant Green's function
}

\author{
F. D. Vila, J. J. Rehr, and J. J. Kas \\ Department of Physics, University of Washington, Seattle, WA 98195 \\ K. Kowalski \\ William R. Wiley Environmental Molecular Sciences Laboratory, Battelle, \\ Pacific Northwest National Laboratory, K8-91, P. O. Box 999, Richland, Washington 99352 \\ B. Peng \\ Physical Sciences Division, Pacific Northwest National Laboratory, Richland, WA 99354
}

(Dated: June 16, 2020)

\begin{abstract}
Green's function methods within many-body perturbation theory provide a general framework for treating electronic correlations in excited states. Here we investigate the cumulant form of the oneelectron Green's function based on the coupled-cluster equation of motion approach in an extension of our previous study. The approach yields a non-perturbative expression for the cumulant in terms of the solution to a set of coupled first order, non-linear differential equations. The method thereby adds non-linear corrections to traditional cumulant methods linear in the self energy. The approach is applied to the core-hole Green's function and illustrated for a number of small molecular systems. For these systems we find that the non-linear contributions lead to significant improvements both for quasiparticle properties such as core-level binding energies, as well as the satellites corresponding to inelastic losses observed in photoemission spectra.
\end{abstract}

\section{INTRODUCTION}

Wavefunction based coupled-cluster (CC) methods have traditionally been used for accurate calculations of ground state electronic correlation effects in molecular systems. ${ }^{1}$ In contrast, Green's function (GF) methods within many-body perturbation theory (MBPT) provide a general formalism for treating electronic correlation effects in excited states. ${ }^{2}$ These effects include the quasiparticle line shape as well as satellites corresponding to the intrinsic inelastic losses due to many-body excitations that are observed in x-ray photoemission spectra (XPS). Recently, a number of CC approaches have been developed for the one-particle GF. For example, a perturbation theoretic approach for the CCGF in frequencyspace has been developed by Peng and Kowalski. ${ }^{3,4}$ As an alternative, our treatment here is based on the equationof-motion coupled-cluster (EOM-CC) approach for the cumulant GF in real-time. Cumulant GF formulations have proved to be advantageous for understanding correlation properties in condensed matter ${ }^{2,5}$ and are exact for model systems with electrons coupled to bosons due to the linked-cluster theorem. ${ }^{6}$ Our initial development was restricted to the treatment of the core-hole GF with an approximate Hamiltonian for a system with a deep core-hole. ${ }^{7}$ Here the approach is extended to include all terms within the CC-singles approximation. This extension yields an exact expression for the cumulant in terms of the solution to a set of coupled first order non-linear differential equations. In particular this approach builds in non-linear terms in the cumulant which significantly improve quantitative calculations. As a quantitative test, the method is applied to a number of small molecular systems. We find that the approach including non-linear corrections yields quasiparticle properties to high accu- racy, as well as an approximate treatment of satellites. Moreover, the approach converges rapidly and gives very good results even at the leading order truncations of the EOM-CC that include non-linear terms.

The structure of this paper is as follows: In Sec II. we first derive an expression for the cumulant in a spinorbital basis in terms of the one-particle self-energy, following the approach of Aryasetiawan et al. ${ }^{8}$ We then show that the 2nd-order approximation for the selfenergy can be derived within the CC approximation using perturbation theory. Next we develop a EOM-CC approach for the cumulant that includes all terms in the CC-singles approximation. We show that this yields a direct, non-perturbative relation between the $\mathrm{CC}$ GF and the exponential cumulant representation in real-time in terms of a set of non-linear differential EOM. Results are then presented in Sec. III. for the quasiparticle properties and satellites in the spectral function for a number of small molecular systems. Finally we present a brief summary and conclusions.

\section{THEORY}

\section{A. Retarded Green's function and cumulant in a spin-orbital basis set}

Within a basis of single-particle spin-orbitals $\left\{\phi_{p}(r)\right\}$ the retarded Green's functions in the time domain is defined as (see SI Sec. I. A.)

$$
G_{p q}\left(t, t^{\prime}\right)=-i \Theta\left(t-t^{\prime}\right)\left\langle 0\left|\left\{a_{p}(t), a_{q}^{\dagger}\left(t^{\prime}\right)\right\}\right| 0\right\rangle,
$$

where the creation and annhilation operators $a_{p}^{\dagger}(t)$ and $a_{q}\left(t^{\prime}\right)$ are associated with spin-orbitals $p$ and $q$ respec- 
tively. Setting $t^{\prime}=0$ for convenience with timedependent operators $O(t)=e^{i H t} O e^{-i H t}$, where $H$ is the full $N$-particle Hamiltonian of the system, we obtain

$$
\begin{array}{r}
G_{p q}(t)=-i \Theta(t)\left[e^{i E_{0} t}\left\langle 0\left|a_{p} e^{-i H t} a_{q}^{\dagger}\right| 0\right\rangle\right. \\
\left.+e^{-i E_{0} t}\left\langle 0\left|a_{q}^{\dagger} e^{i H t} a_{p}\right| 0\right\rangle\right],
\end{array}
$$

where $E_{0}$ is the total energy of the ground state $|0\rangle$, which is the eigenvalue of $H|0\rangle=E_{0}|0\rangle$. From Eq. (2) we obtain the Green's function of the non-interacting system $G_{p q}^{0}(t)$ using the relations

$$
\begin{aligned}
H & \rightarrow H_{0}=\sum_{p} \epsilon_{p} a_{p}^{\dagger} a_{p} \\
|0\rangle & \rightarrow|\Phi\rangle \\
E_{0} & \rightarrow E_{0}^{0}=\left\langle\Phi\left|H_{0}\right| \Phi\right\rangle=\sum_{i} \epsilon_{i} .
\end{aligned}
$$

Here and below we use the convention where $i, j, k, \ldots$ correspond to occupied ("hole") states in the HartreeFock single-determinant $|\Phi\rangle, a, b, c, \ldots$ indices correspond to unoccupied ("particle") states, and $p, q, r, \ldots$ can be either. With these definitions,

$$
G_{p q}^{0}(t)=-i \Theta(t) e^{-i \epsilon_{p} t} \delta_{p q} .
$$

The cumulant ansatz for the Green's function in the timedomain for a given one-particle orbital $p$ is defined ${ }^{8,9}$ by the exponential representation

$$
G_{p}(t)=G_{p}^{0}(t) e^{C_{p}(t)} .
$$

Here $G_{p}(t)$ and $G_{p}^{0}(t)$ are the interacting and noninteracting retarded Green's functions for one-particle orbital $p$, respectively, and $C_{p}(t)$ is the retarded cumulant. Eq. (5) can be generalized to its non-diagonal, spinorbital matrix form similar to Eq. (1) (see SI Sec. I. A.): $\hat{G}(t)=\hat{G}^{0}(t) e^{\hat{C}(t)}$, where $\hat{G}, \hat{G}^{0}$ and $\hat{C}$ are the matrix forms of the Green's functions and cumulant respectively. In the conventional formulation based on the decoupling approximation, the cumulant is obtained by matching the cumulant expansion of $G_{p}(t)$ to first order with that from the Dyson equation $G=G^{0}+G^{0} \Sigma G$, (see SI Sec. I. B.). ${ }^{8,9}$ Since $\hat{G}^{0}$ is diagonal in the one-particle HF eigenstates $p$, the cumulant satisfies the relation

$$
G_{p p}^{0}(t) C_{p q}(t)=\int d t_{1} d t_{2} G_{p p}^{0}\left(t-t_{1}\right) \Sigma_{p q}\left(t_{1}-t_{2}\right) G_{q q}^{0}\left(t_{2}\right)
$$

Introducing the Fourier transform of the double convolution in the right hand term and the form of $\hat{G}^{0}$ from Eq. (4) we obtain for $t \geq 0$

$$
C_{p q}(t)=i \int \frac{d \omega}{2 \pi} e^{i\left(\omega+\epsilon_{p}\right) t} G_{p p}^{0}(\omega) \Sigma_{p q}(\omega) G_{q q}^{0}(\omega)
$$

Then shifting the $\omega$ integration variable in the Fourier transform with $G_{p p}^{0}(\omega)=\left(\omega-\epsilon_{p}+i \delta\right)^{-1}$, and approximating the self-energy with its diagonal form $\Sigma_{p q}(\omega) \simeq$
$\Sigma_{p p}(\omega) \delta_{p q}$, the diagonal elements of the cumulant become

$$
C_{p p}(t)=\int \frac{d \omega}{2 \pi} \frac{i \Sigma_{p p}\left(\omega+\epsilon_{p}\right)}{(\omega+i \delta)^{2}} e^{-i \omega t} .
$$

Finally since the matrix exponential is now diagonal

$$
G_{p p}(t)=-i \Theta(t) e^{-i \epsilon_{p} t+C_{p p}(t)},
$$

which is the standard form of the cumulant Green's function for the diagonal elements.

\section{B. Second order approximations}

As shown above, the cumulant to lowest order is linear in the one-particle self-energy, so the problem reduces to finding a suitable approximation for $\Sigma_{p p}(\omega)$. In the GW approximation of Hedin, ${ }^{2}$ for example, $\Sigma=i G W$ is approximated to first order in the screened electronelectron interaction $W=\epsilon^{-1} V$, where $\epsilon$ is the dielectric function. Here, following the usual practice for molecular systems, screening is neglected, and hence to leading order $\Sigma=\Sigma^{(2)}$ becomes the 2nd-order self-energy (SE2). ${ }^{10,11}$ We also show that $\Sigma^{(2)}$ can obtained using the perturbation theory and the $\mathrm{CC}$ approximation with, at least doubles, to lowest non-vanishing order.

\section{Second order self-energy and cumulant}

Within MBPT and assuming a single-determinant Hartree-Fock reference $|\Phi\rangle$, the 2nd-order self-energy in the spin-orbital basis is given by ${ }^{10,11}$

$$
\begin{aligned}
\Sigma_{p q}^{(2)}(\omega)= & \frac{1}{2} \sum_{i a b} \frac{\langle p i \| a b\rangle\langle a b \| q i\rangle}{\omega+\epsilon_{i}-\epsilon_{a}-\epsilon_{b}}+ \\
& +\frac{1}{2} \sum_{i j a} \frac{\langle p a \| i j\rangle\langle i j \| q a\rangle}{\omega+\epsilon_{a}-\epsilon_{i}-\epsilon_{j}}
\end{aligned}
$$

where $\langle p q \| r s\rangle=\langle p q \mid r s\rangle-\langle p q \mid s r\rangle$ are the antisymmetric Coulomb integrals over the real $p, q, r, s$ spin-orbitals. The diagonal terms in Eq. (8) can be written as

$$
\Sigma_{p p}^{(2)}\left(\omega+\epsilon_{p}\right)=\frac{1}{2} \sum_{i a b} \frac{\langle p i \| a b\rangle^{2}}{\omega-\epsilon_{p i}^{a b}}+\frac{1}{2} \sum_{i j a} \frac{\langle p a \| i j\rangle^{2}}{\omega-\epsilon_{p a}^{i j}},
$$

where $\epsilon_{p i}^{a b}=\epsilon_{a}+\epsilon_{b}-\epsilon_{p}-\epsilon_{i}$ and $\epsilon_{p a}^{i j}=\epsilon_{i}+\epsilon_{j}-\epsilon_{p}-$ $\epsilon_{a}$. Consequently to 2 nd-order in perturbation theory the 2nd order cumulant is obtained with $\Sigma_{p p} \simeq \Sigma_{p p}^{(2)}$ in Eq. (8)

$$
\begin{aligned}
C_{p p}^{(2)}(t) & =\frac{1}{2} \sum_{i a b}\langle p i \| a b\rangle^{2} \int \frac{d \omega}{2 \pi} \frac{i e^{-i \omega t}}{\omega^{2}\left(\omega-\epsilon_{p i}^{a b}\right)}+ \\
& +\frac{1}{2} \sum_{i j a}\langle p a \| i j\rangle^{2} \int \frac{d \omega}{2 \pi} \frac{i e^{-i \omega t}}{\omega^{2}\left(\omega-\epsilon_{p a}^{i j}\right)} .
\end{aligned}
$$


Then using the identity

$$
\int \frac{d \omega}{2 \pi} \frac{i e^{-i \omega t}}{\omega^{2}(\omega-\epsilon)}=\frac{1}{\epsilon^{2}}\left(e^{-i \epsilon t}+i \epsilon t-1\right) \operatorname{sgn}(t)
$$

the 2nd-order cumulant can be expressed as

$$
\begin{aligned}
C_{p p}^{(2)}(t)= & \frac{1}{2} \sum_{i a b}\left(u_{i p}^{a b}\right)^{2}\left(e^{-i \epsilon_{p i}^{a b} t}+i \epsilon_{p i}^{a b} t-1\right)+ \\
& +\frac{1}{2} \sum_{i j a}\left(u_{p a}^{i j}\right)^{2}\left(e^{-i \epsilon_{p a}^{i j} t}+i \epsilon_{p a}^{i j} t-1\right)
\end{aligned}
$$

where the cumulant amplitudes $u_{p q}^{r s}$ are

$$
u_{p q}^{r s}=\frac{\langle p q \| r s\rangle}{\epsilon_{p q}^{r s}} .
$$

For an occupied core state $p$, the $u_{i p}^{a b}$ coefficients are equivalent to the doubly-excited CC amplitudes approximated to 1st order in Moller-Plesset MBPT. ${ }^{12}$ This result is equivalent to the first iteration in the solution of any $\mathrm{CC}$ formulation that includes $\mathrm{T}_{2}$ (when the initial guess is the null vector) ${ }^{12}$ thus demonstrating a direct connection between the exponential form of the retarded cumulant and the CC approach. The behavior of the cumulant for the 2nd-order self energy is similar to that for electrons coupled to bosonic excitations labeled by an index $q$ in the quasi-boson approximation with coupling coefficients $g_{q},{ }^{2}$

$$
C_{p p}^{(2)}(t)=\sum_{q} \frac{g_{q}^{2}}{\omega_{q}^{2}}\left(e^{-i \omega_{q} t}+i \omega_{q} t-1\right)
$$

where $g_{q}=\omega_{q} u_{q}$.

For analysis purposes, it is convenient to define a cumulant kernel $\beta(\omega)$ that characterizes the spectrum of excitations

$$
\beta(\omega)=\sum_{q} g_{q}^{2} \delta\left(\omega-\omega_{q}\right),
$$

where for the SE2,

$$
\beta(\omega)=-\frac{1}{\pi} \operatorname{Im} \Sigma_{p p}^{(2)}\left(\omega+\epsilon_{p}\right) .
$$

Thus $\beta(\omega)$ is given by the poles of the $\Sigma_{p p}^{(2)}$. As a consequence the cumulant can also be defined by the kernel $\beta(\omega)$

$$
C_{p p}^{(2)}(t)=\int d \omega \frac{\beta(\omega)}{\omega^{2}}\left(e^{-i \omega t}+i \omega t-1\right) .
$$

This expression is referred to as the Landau form of the cumulant, and facilitates the interpretation of excitations in the spectrum. For example, since $C_{p p}^{(2)}(0)=C_{p p}^{(2)^{\prime}}(0)=$ 0 , the Landau form guarantees that the spectral function $A_{p p}(\omega)=(-1 / \pi) \operatorname{Im} G_{p p}(\omega)$ is normalized and has an invariant centroid at the independent particle energy $\epsilon_{p}$ as in Koopmans' theorem.

\section{2nd-order CC Green's function}

It is interesting to note that 2 nd-order perturbation theory for $G_{p q}^{R}(\omega)$ based on the CC ansatz and including at least $\mathrm{T}_{2}$ double excitations yields the same 2 nd-order self-energy $\Sigma^{(2)}(\omega)$ discussed above. Here we demonstrate this equivalence in the frequency domain, since this treatment avoids the complication of expanding the exponential propagation operator in Eq. (2) in successive orders of perturbation theory. We start with the frequency domain version of the GF corresponding to Eq. (2):

$$
\begin{aligned}
G_{p q}^{R}(\omega)= & \left\langle\Phi\left|(1+\Lambda) \overline{a_{q}^{\dagger}}\left(\omega+\bar{H}_{N}+i \delta\right)^{-1} \overline{a_{p}}\right| \Phi\right\rangle+ \\
& \left\langle\Phi\left|(1+\Lambda) \overline{a_{p}}\left(\omega-\bar{H}_{N}+i \delta\right)^{-1} \overline{a_{q}^{\dagger}}\right| \Phi\right\rangle
\end{aligned}
$$

equivalent to the CC GF (see SI Sec. I C) in Eq. (17) of Ref. 13, but including both the $N-1$ and $N+1$ branches. We include the $N+1$ branch for completeness, although in the cases studied here it only has a small contribution to the total GF. In Eq. 20, $|\Phi\rangle$ is the reference HF determinant, $\bar{O}=e^{-T} O e^{T}$ is the similarity transformed $O$ operator, $H_{N}$ is the normal ordered Hamiltonian, $\Lambda$ is the $\mathrm{CC}$ de-excitation operator, and we have used the Baker-Campbell-Hausdorff $(\mathrm{BCH})$ relation

$$
\begin{aligned}
\bar{a}_{p} & =a_{p}+\left[a_{p}, T\right] \\
\overline{a_{q}^{\dagger}} & =a_{q}^{\dagger}+\left[a_{q}^{\dagger}, T\right] .
\end{aligned}
$$

Inserting the retarded form of the auxiliary $N-1$ operator $X_{p}(\omega)=\left(\omega+\bar{H}_{N}+i \delta\right)^{-1} \bar{a}_{p}$ and the $N+1$ operator $Y_{q}(\omega)=\left(\omega-\bar{H}_{N}+i \delta\right)^{-1} \overline{a_{q}^{\dagger}}$ into Eq. (20)

$$
\begin{aligned}
G_{p q}^{R}(\omega)= & \left\langle\Phi\left|(1+\Lambda) \overline{a_{q}^{\dagger}} X_{p}(\omega)\right| \Phi\right\rangle+ \\
& \left\langle\Phi\left|(1+\Lambda) \bar{a}_{p} Y_{q}(\omega)\right| \Phi\right\rangle .
\end{aligned}
$$

If we now assume that $T=T_{2}=\frac{1}{4} \sum_{i j a b} t_{i j}^{a b} a_{a}^{\dagger} a_{b}^{\dagger} a_{j} a_{i}$ and $\Lambda=\Lambda_{2}=\frac{1}{4} \sum_{i j a b} \lambda_{i j}^{a b} a_{i}^{\dagger} a_{j}^{\dagger} a_{b} a_{a}$, and expand all the operators to 2nd-order and simplify (see SI Sec. I D)

$$
\begin{aligned}
G_{p q}^{R(2)}(\omega)= & \frac{\delta_{p q}}{\left(\omega-\epsilon_{p}\right)}+\frac{1}{\left(\omega-\epsilon_{p}\right)} \times \\
& \times\left[\frac{1}{2} \sum_{i j a} \frac{v_{i j}^{q a} v_{i j}^{p a}}{\left(\omega+\epsilon_{a}-\epsilon_{i}-\epsilon_{j}\right)}+\right. \\
& \left.+\frac{1}{2} \sum_{i a b} \frac{v_{a b}^{p i} v_{a b}^{q i}}{\left(\omega+\epsilon_{i}-\epsilon_{a}-\epsilon_{b}\right)}\right] \frac{1}{\left(\omega-\epsilon_{q}\right)} .
\end{aligned}
$$

Note that this 2nd-order result for Green's function has the form of a Dyson equation $\hat{G}^{R(2)}(\omega)=\hat{G}_{0}^{R}(\omega)+$ $\hat{G}_{0}^{R}(\omega) \hat{\Sigma}^{(2)}(\omega) \hat{G}_{0}^{R(2)}(\omega)$, where the expression in brackets is identical to the 2nd-order self-energy of Eq. (10). Consequently the Fourier transform of Eq. (23) yields the same 2nd-order cumulant as Eq. (8). 


\section{Real-time EOM-CC Cumulant GF}

In order to explore corrections to the 2nd-order approximation for the cumulant GF, we now develop a realtime approximation based on the more general equation of motion (EOM-CC) ansatz. Our treatment here extends that introduced in our original approach ${ }^{7}$ by including terms up to third order in the CC amplitudes, and gives a non-perturbative representation for the $\mathrm{cu}-$ mulant. For definiteness, we restrict our discussion here to the retarded core-hole Green's function for a given deep core level $p=c, G_{c}^{R}=G_{c c}$ given by

$$
\begin{aligned}
G_{c}^{R}(t)= & -i \Theta(t) e^{i E_{0} t}\left\langle 0\left|a_{c} e^{-i H t} a_{c}^{\dagger}\right| 0\right\rangle+ \\
& -i \Theta(t) e^{-i E_{0} t}\left\langle 0\left|a_{c}^{\dagger} e^{i H t} a_{c}\right| 0\right\rangle .
\end{aligned}
$$

We then introduce the separable approximation to the ground state $|0\rangle \simeq a_{c}^{\dagger}|N-1\rangle$, where $|N-1\rangle$ is the fully correlated $N-1$ electron part of the $N$ electron wavefunction with the core electron separated from it. Inserting this into Eq. (24) and remembering that $a_{c} a_{c}=0$,

$$
G_{c}^{R}(t)=-i \Theta(t) e^{-i E_{0} t}\left\langle N-1\left|e^{i H t}\right| N-1\right\rangle .
$$

Formally $|N-1, t\rangle=e^{i H t}|N-1\rangle$ is a solution to

$$
-i \frac{d|N-1, t\rangle}{d t}=H|N-1, t\rangle
$$

so that

$$
G_{c}^{R}(t)=-i \Theta(t) e^{-i E_{0} t}\langle N-1 \mid N-1, t\rangle .
$$

Next we assume a time-dependent, CC ansatz for $|N-1, t\rangle=N(t) e^{T(t)}|\phi\rangle$. It is important to note that the excitation operator $T$ acts in the $N-1$ particle Fock space, rather than in the $N$ space as in standard ground-state $\mathrm{CC}$, and that the reference determinant is $|\phi\rangle=a_{c}|\Phi\rangle$, where $|\Phi\rangle$ is the $N$ electron HF determinant of the ground state. Thus, our treatment here refers to a CC approximation to the excited states involved in the calculation of the Green's function, rather that the typical applications where the CC ansatz is used for the ground state. Inserting this ansatz into the differential equation for $|N-1, t\rangle$ and left multiplying by $e^{-T(t)}$, we obtain the coupled EOM

$$
-i\left[\frac{d \ln N(t)}{d t}+\frac{d T(t)}{d t}\right]=\bar{H}(t)|\phi\rangle,
$$

where the similarity transformed Hamiltonian is $\bar{H}(t)=$ $e^{-T(t)} H e^{-T(t)}$. Here in order to simplify both the notation and the computation of the matrix elements, instead of the exact second-quantized Hamiltonian

$$
H=\sum_{p q} h_{p q} a_{p}^{\dagger} a_{q}+\frac{1}{4} \sum_{p q r s} v_{p q}^{r s} a_{p}^{\dagger} a_{q}^{\dagger} a_{s} a_{r}
$$

where $h_{p q}$ are the single particle kinetic and electronnuclei molecular orbital integrals, we introduce its normal ordered form $H_{N}=H-\langle\phi|H| \phi\rangle$. The similarity transformed form of $H_{N}$ is $\bar{H}_{N}(t)=\bar{H}(t)-E^{N-1}$, where $E^{N-1}=\langle\phi|\bar{H}| \phi\rangle$. It should be noted that with an $N-1$ reference, $H_{N}$ no longer has the usual diagonal single particle term but rather

$$
H_{N}=\sum_{p q} f_{p q}\left\{a_{p}^{\dagger} a_{q}\right\}^{\prime}+\frac{1}{4} \sum_{p q r s} v_{p q}^{r s}\left\{a_{p}^{\dagger} a_{q}^{\dagger} a_{s} a_{r}\right\}^{\prime}
$$

where the \{\}$^{\prime}$ is a reminder that the normal ordering is done with respect to the $N-1$ particle reference $|\phi\rangle$, and $f_{p q}=\epsilon_{p} \delta_{p q}-v_{p c}^{q c}$. We now follow the usual CC approach of projecting Eq. (28) from the left with reference $\langle\phi|$ and the $i \rightarrow a, j \rightarrow b, \ldots$ excited reference $\left\langle\phi_{i j \ldots}^{a b \ldots}\right|$ to separate the EOM for $N(t)$ and $T(t)$,

$$
\begin{array}{r}
-i \frac{d \ln N(t)}{d t}=\left\langle\phi\left|\bar{H}_{N}(t)\right| \phi\right\rangle+E^{N-1}, \\
-i\left\langle\phi_{i j \ldots}^{a b \ldots}\left|\frac{d T(t)}{d t}\right| \phi\right\rangle=\left\langle\phi_{i j \ldots}^{a b \ldots}\left|\bar{H}_{N}(t)\right| \phi\right\rangle .
\end{array}
$$

It is interesting to note that these equations have a structure identical to the standard CC equations for the ground state, ${ }^{12}$ except that now we are interested in $N(t)$ and $T(t)$ instead of $E_{C C}$ and $T$. Moreover, the equations are now non-linear coupled first order differential equations rather than algebraic equations. The only matrix elements required to get explicit expressions are $\left\langle\phi\left|\bar{H}_{N}(t)\right| \phi\right\rangle,\left\langle\phi_{i j \ldots}^{a b \ldots}|d T(t) / d t| \phi\right\rangle$, and $\left\langle\phi_{i j \ldots}^{a b \ldots}\left|\bar{H}_{N}(t)\right| \phi\right\rangle$. In order to evaluate these results, we need to introduce some further approximations. First, we assume that the ground state is uncorrelated, i.e. $|N-1\rangle \simeq a_{c}|\Phi\rangle=|\phi\rangle$, so that

$$
\begin{aligned}
\langle N-1 \mid N-1, t\rangle & =N(t)\left\langle N-1\left|e^{i H t}\right| \phi\right\rangle \\
& =N(t)\left\langle\phi\left|e^{i H t}\right| \phi\right\rangle \\
& =N(t)(1+\langle\phi|R(t)| \phi\rangle) \\
& =N(t) .
\end{aligned}
$$

Here $R(t)$ is the excitation operator that collects all excited terms arising from the series expansion of $e^{i H t}$ and has expectation value $\langle\phi|R(t)| \phi\rangle=0$ due to orthogonality. The approximation of an uncorrelated ground state could be relaxed by replacing $|\Phi\rangle$ by $\exp (T)|\Phi\rangle$. However, with the approximation introduced above, the core-hole Green's function $G_{c}^{R} t$ ) is directly related to the normalization factor $N(t)$

$$
G_{c}^{R}(t)=-i \Theta(t) e^{-i E_{0} t} N(t) .
$$

We note that the normalization factor $N(t)$ also corresponds to the vacuum fluctuations in field theory treatments. ${ }^{14}$ Given that no correlation is included in the ground state, we can simply approximate $E_{0} \simeq E_{\mathrm{HF}}$, i.e., the HF energy of the $N$-particle system, and hence $E_{0}-E^{N-1} \simeq \epsilon_{c}$, as expected from Koopmans' theorem. 
The logarithmic derivative in the EOM in Eq. (31) implies that $N(t)$ is a pure exponential, so $G_{c}^{R}(t)$ has an explicit cumulant form in the time-domain

$$
G_{c}^{R}(t)=-i \Theta(t) e^{-i \epsilon_{c} t} e^{C_{c}^{R}(t)},
$$

where the cumulant is obtained by integrating Eq. (31)

$$
C_{c}^{R}(t)=i \int_{0}^{t}\left\langle\phi\left|\bar{H}_{N}(t)\right| \phi\right\rangle d t^{\prime}
$$

with the boundary condition $C_{c}^{R}(0)=0$. We then make one further approximation for the treatment here, namely that the operator $T$ is restricted to single excitations $T(t)=T_{1}(t) \equiv \sum_{i a} t_{i}^{a}(t)\left\{a_{a}^{\dagger} a_{i}\right\}^{\prime}$, where again the \{\}$^{\prime}$ make explicit that the contractions are with respect to that $N-1$ reference. Thus the occupied indices $i, j, \ldots$ do not include the the core index $c$, though the unoccupied $a, b, \ldots$ levels do. This will be assumed implicitly in all sums below. We also suppress the time-dependence label in the CC amplitudes $t_{i}^{a}(t)$ unless needed for clarity. In order to obtain explicit expressions for the amplitudes within this $T_{1}$ approximation we need to calculate the matrix elements $\left\langle\phi\left|\bar{H}_{N}(t)\right| \phi\right\rangle,\left\langle\phi_{i}^{a}|d T(t) / d t| \phi\right\rangle$, and $\left\langle\phi_{i}^{a}\left|\bar{H}_{N}(t)\right| \phi\right\rangle$. To begin, we note that although the full similarity transformed Hamiltonian is given by

$$
\begin{gathered}
\bar{H}_{N}(t)=H_{N}+\left(H_{N} T_{1}(t)\right)_{c}+\frac{1}{2 !}\left(H_{N} T_{1}(t)^{2}\right)_{c}+ \\
\frac{1}{3 !}\left(H_{N} T_{1}(t)^{3}\right)_{c}+\frac{1}{4 !}\left(H_{N} T_{1}(t)^{4}\right)_{c},
\end{gathered}
$$

the quartic terms do not contribute to the matrix elements of interest. After some straightforward, though tedious algebra and diagrammatic analysis, we obtain a compact expression for Eq. (31)

$$
\begin{aligned}
-i \frac{d C_{c}^{R}(t)}{d t} & =\left\langle\phi\left|\bar{H}_{N}(t)\right| \phi\right\rangle \\
& =\sum_{i a} f_{i a} t_{i}^{a}+\frac{1}{2} \sum_{i j a b} v_{i j}^{a b} t_{j}^{b} t_{i}^{a},
\end{aligned}
$$

The EOM of the CC amplitudes are

$$
-i \dot{t}_{i}^{a}=\left\langle\phi_{i}^{a}\left|\frac{d T(t)}{d t}\right| \phi\right\rangle=\left\langle\phi_{i}^{a}\left|\bar{H}_{N}(t)\right| \phi\right\rangle,
$$

with boundary conditions $t_{i}^{a}(0)=0$, where the matrix elements are obtained from expressions with matrix products up to third order in the CC amplitudes,

$$
\begin{aligned}
\left\langle\phi_{i}^{a}\left|\bar{H}_{N}(t)\right| \phi\right\rangle & =f_{a i}+\sum_{b} f_{a b} t_{i}^{b}-\sum_{j} f_{j i} t_{j}^{a} \\
& +\sum_{j b} v_{a j}^{i b} t_{j}^{b}-\sum_{j b} f_{j b} t_{i}^{b} t_{j}^{a} \\
& -\sum_{j k b} v_{i b}^{j k} t_{j}^{a} t_{k}^{b}+\sum_{j b c} v_{a j}^{b c} t_{i}^{b} t_{j}^{c} \\
& -\sum_{j k b d} v_{j k}^{b d} t_{i}^{b} t_{j}^{a} t_{k}^{d} .
\end{aligned}
$$

It is important to note that these matrix elements are analogous to those obtained with the standard CCSD approximation to the ground state when only singles are included (i.e., $T_{2}=0$ ).

We can now introduce the explicit forms of the $f_{p q}$ elements to make the results in Eq. (38) and (40) more explicit. First the exact form of the cumulant in Eq. (35) is defined by the matrix element $\left\langle\phi\left|\bar{H}_{N}(t)\right| \phi\right\rangle$ and given by the compact expression with terms linear $(\mathrm{L})$ and non-linear (NL) in the amplitudes $t_{i}^{a}$ and first order in the couplings $v_{i j}^{a b}$,

$$
\left\langle\phi\left|\bar{H}_{N}(t)\right| \phi\right\rangle=-\sum_{i a} v_{c i}^{c a} t_{i}^{a}+\frac{1}{2} \sum_{i j a b} v_{i j}^{a b} t_{i}^{a} t_{j}^{b} .
$$

The linear (L) term corresponds to the coupling between the core-hole and the particle-hole excitation $i \rightarrow a$, while the quadratic terms (NL) represent valence polarization effects that characterize the screening of the the corehole. Similarly the matrix elements for the EOM of the $\mathrm{CC}$ amplitudes are

$$
\begin{aligned}
\left\langle\phi_{i}^{a}\left|\bar{H}_{N}(t)\right| \phi\right\rangle & =-v_{a c}^{i c}+\left(\epsilon_{a}-\epsilon_{i}\right) t_{i}^{a} \\
& +\sum_{j} v_{j c}^{i c} t_{j}^{a}-\sum_{b} v_{a c}^{b c} t_{i}^{b}+\sum_{j b} v_{j a}^{b i} t_{j}^{b} \\
& +\sum_{j b} v_{j c}^{b c} t_{i}^{b} t_{j}^{a}+\sum_{j b d} v_{a j}^{b d} t_{i}^{b} t_{j}^{d}-\sum_{j k b} v_{j k}^{i b} t_{j}^{a} t_{k}^{b} \\
& -\sum_{j k b d} v_{j k}^{b d} t_{i}^{b} t_{j}^{a} t_{k}^{d} .
\end{aligned}
$$

Note that the exponential form of Eq. (35) is identical to that in our original paper. ${ }^{7}$ However, the differential equations for the $\mathrm{CC}$ coefficients have terms up to third order. Note also that if one keeps only the first two terms on the RHS of Eq. (42), the cumulant becomes that for the 2nd-order self energy in Eq. (12). As discussed below, however, the non-linear term turn out to be crucial for accurate calculations.

The result for this more general EOM-CC cumulant can also be represented in Landau form with a cumulant kernel $\beta(\omega)$ given by

$$
\beta(\omega)=\frac{1}{\pi} \operatorname{Re} \int_{0}^{\infty} d t e^{-i \omega t} \frac{d}{d t}\left\langle\phi\left|\bar{H}_{N}(t)\right| \phi\right\rangle .
$$

In contrast to the expression in terms of the 2nd-order self-energy, the general EOM-CC kernel $\beta(\omega)$ implicitly contains non-linear terms that give corrections to 2 ndorder approximations in the SE2 or GW formulations. As a consequence $\beta(\omega)$ is no longer guaranteed to be positive definite, and similarly the spectral function no longer has multiple-satellites, consistent with the the particle-hole nature of the excitations. ${ }^{15}$ Finally we also note that a one-particle form of Eq. (40) for the CC amplitudes can lead to simpler methods based on alternative one-body, effective Hamiltonians (see SI Sec. I. E.). 


\section{RESULTS}

\section{A. Computational Details}

In this section we illustrate our approach for calculations of the spectral function for the ten electron (10e) series systems: $\mathrm{CH}_{4}, \mathrm{NH}_{3}, \mathrm{H}_{2} \mathrm{O}$, $\mathrm{HF}$ and $\mathrm{Ne}$, using different levels of approximation to the EOM-CCS approach and cumulant, as well as different basis sets. Our calculations use experimental geometries ${ }^{16}$ for all the molecules: $r_{\mathrm{CH}}=1.087 \AA, r_{\mathrm{NH}}=1.012 \AA, a_{\mathrm{HNH}}=106.67^{\circ}, r_{\mathrm{OH}}=$ $0.958 \AA, a_{\mathrm{HOH}}=104.48^{\circ}, r_{\mathrm{FH}}=0.917 \AA$. To assess the effect of basis set quality, calculations were performed with the DZVP, ${ }^{17}$ cc-pVDZ, ${ }^{18}$ and aug-cc-pVDZ ${ }^{19}$ basis sets. The parameters for the KT, DSE2, CSE2 and EOM-CC methods, the molecular orbital energies $\epsilon_{p}$ and integrals $v_{p q}^{r s}$, were computed using PyQuante. ${ }^{17}$ To improve the efficiency of these methods (in particular in the case of the EOM-CC method), only integrals greater than $1 \times 10^{-4}$ au were used, i.e., the integrals were screened after the SCF was properly converged. As shown in SI Sec. II. A., this approximation greatly reduces the amount of data that needs to be handled and results in faster simulations (by an order of magnitude in some cases), with little effect on the calculated spectral functions. The integrals were not screened for the GFCCSD and GFCC-i $(2,3)$ calculations. The time integration of the EOM in Eq. (39) was performed using a 4th-order Adams-Moulton linear multistep method. ${ }^{20}$ To obtain the sharp, broad energy range and smooth spectral functions shown below, the integration used a time step of 0.025 au $(\sim 0.6$ as) with a total simulation time of 600 au $(\sim 14.5 \mathrm{fs})$.

\section{B. Levels of Approximation}

The results presented below use four levels of approximation for the EOM-CCS calculations from Eq. (35)-(42) beyond the independent particle approximation (Koopmans' theorem):

0. Second-order approximation obtained by keeping only the first two terms on the RHS of Eq. (42); this yields a cumulant GF identical to that obtained with the 2nd-order self energy (CSE2).

1. Core approximation obtained by keeping the terms in 0 plus the dominant corrections to the 2 nd-order approximation, i.e., the first four sums in Eq. (42); this includes all linear valence-valence sums plus the quadratic term from excitations coupled to the core-hole.

2. Quadratic approximation which includes terms in 1 plus the fifth and sixth sums in Eq. (42) corresponding to quadratic valence-valence terms; these new terms give corrections that shift the excitation energies closer to the QP peak.
3. Full $\mathrm{T}_{1}$ approach obtained by keeping all terms in the EOM-CCS approximation in Eq. (42), including the third order term in the $\mathrm{CC}$ amplitudes in the last line of Eq. (42).

Each of this approximations to Eq. (42) can be paired with linear (L) and non-linear (NL) approximations to the cumulant defined in Eq. (36), which we label as $0_{L}$, $0_{N L}, 1_{L}, 1_{N L}$, etc.

For comparison we also include results for i) the bare energy or Koopmans' Theorem (KT); ii) the exact solution of the Dyson equation using the diagonal 2nd-order self energy (DSE2)

$$
G_{p}(\omega)=\left[1-G_{p}^{0}(\omega) \Sigma_{p p}^{(2)}(\omega)\right]^{-1} G_{p}^{0}(\omega)
$$

and iii) results from the GFCCSD and GFCC-i $(2,3)$ methods (using only the cc-pVDZ and aug-cc-pVDZ basis sets). ${ }^{4,21,22}$

\section{Quasiparticle properties}

Table I presents a comparison between the experimental core binding energies of the $10 e$ systems to those computed with the aug-cc-pVDZ basis set. We also include KT, DSE2, GFCCSD and GFCC-i $(2,3)$ results. Equivalent tables for the other basis sets are given in the SI. While KT seriously overestimates the core-binding energies the GFCC results (including both GFCCSD and GFCC-i $(2,3))$ give significant improvements. Notably the inclusion of inner triples in the $\operatorname{GFCC}-\mathrm{i}(2,3)$ reduces the MAE from $4.24 \mathrm{eV}$ to $2.83 \mathrm{eV}$. The results from the EOM-CC approach with only the linear (L) approximation for the cumulant are comparable, with slightly larger MAEs from 4.75 to $5.28 \mathrm{eV}$. Surprisingly the DSE2 results for the quasiparticle peak are slightly better, with absolute discrepancies of up to $2 \mathrm{eV}$ and a MAE slightly over $1 \mathrm{eV}$ over a broad energy regime.

The quality of the EOM-CCS results depends primarily on the inclusion of non-linear terms in the cumulant rather than level of approximation used for the $\mathrm{CC}$ amplitudes in Eq. (39), so long as the non-linear terms are included. The linear (L) approximation to the cumulant from the first term on the right in Eq. (41) consistently underestimates the binding energy by about $3-6$ $\mathrm{eV}$. Notably, the introduction of the NL terms in Eq. (41) reduces the error by an order of magnitude, thereby bringing the results for the binding energy in very good agreement with experiment, with mean absolute errors (MAE) of $0.7 \mathrm{eV}$ or less. Remarkably all three non-linear approximations to the EOM-CCS equations (i.e., levels $\left.1_{N L}-3_{N L}\right)$, produce similar errors, with a systematic underestimation of the experimental results by less than an $\mathrm{eV}$. However, the full $\left(3_{N L}\right)$ treatment of the $\mathrm{T}_{1}$ term does not improve the trend. This suggests that terms beyond the CC-singles approximation in Eqs. (31) and (32) are desirable in an effort to achieve higher accuracy. Nevertheless, our EOM-CC cumulant results demonstrate 
TABLE I: Comparison of the experimental core binding energies (in eV) to those obtained with the aug-cc-pVDZ basis set, using the L and NL approximations to the cumulant and the 1-3 approximations of the EOM-CCS method, and their mean absolute errors (MAE). The results from GFCCSD and GFCC-i $(2,3)$ are obtained from the coupled-cluster Green's function approaches $^{3,4}$ and improve on the cumulant with only linear terms in the CC coefficients. However, the inclusion of the non-linear terms in the cumulant significantly improves the agreement with experiment.

\begin{tabular}{|c|c|c|c|c|c|c|c|c|c|c|c|c|}
\hline System & $\mathrm{KT}$ & DSE2 & GFCCSD & GFCC-i $(2,3)$ & $\mathbf{1}_{L}$ & $\mathbf{2}_{L}$ & $\mathbf{3}_{L}$ & $\mathbf{1}_{N L}$ & $\mathbf{2}_{N L}$ & $\mathbf{3}_{N L}$ & Expt & Ref \\
\hline $\mathrm{CH}_{4}$ & 305.18 & 292.24 & 293.34 & 292.69 & 286.35 & 287.31 & 286.89 & 290.020 & 290.62 & 290.36 & 290.703 & {$[23]$} \\
\hline $\mathrm{NH}_{3}$ & 423.18 & 405.93 & 409.10 & 407.90 & 400.18 & 400.85 & 400.25 & 404.865 & 405.27 & 404.92 & 405.52 & {$[24]$} \\
\hline $\mathrm{H}_{2} \mathrm{O}$ & 559.91 & 538.97 & 544.28 & 542.76 & 534.15 & 534.23 & 533.56 & 539.225 & 539.28 & 538.89 & 539.7 & {$[25]$} \\
\hline $\mathrm{HF}$ & 715.89 & 692.29 & 699.39 & 697.48 & 688.91 & 688.40 & 687.81 & 693.710 & 693.40 & 693.03 & 694.2 & {$[26]$} \\
\hline $\mathrm{Ne}$ & 892.40 & 868.15 & 875.44 & 873.70 & 866.60 & 865.80 & 865.44 & 870.458 & 869.91 & 869.66 & 870.2 & $27]$ \\
\hline MAE & 19.25 & 1.33 & 4.24 & 2.83 & 4.83 & 4.75 & 5.28 & 0.51 & 0.37 & 0.69 & & \\
\hline
\end{tabular}

that even the simplest $T_{1}$ approximation is capable of recovering most of the relaxation energy required to reproduce experimental quasiparticle binding energies to within an $\mathrm{eV}$. This result is consistent with the typically very good relaxation energies obtained with $\triangle \mathrm{SCF}$ approaches. These can be cast in terms of $e^{T_{1}}$ rotations of the ground state orbitals, as demonstrated by Thouless' theorem. Finally, for the EOM-CCS method all three basis sets used here yield about the same MAE, showing that the quasiparticle energy can be computed to within $1 \mathrm{eV}$ even with rather modest basis sets.

Table II shows a comparison between the quasiparticle strengths (i.e., the renormalization constants) for the $10 e$ systems computed with the best basis set (aug-ccpVDZ) using the L and NL approximations to the cumulant and the 1-3 approximations of the EOM-CCS method. The two approximations to the cumulant show similar trends, with the strength increasing almost systematically in the $\mathrm{CH}_{4}-\mathrm{Ne}$ series. The DSE2 results do not show this trend, and are systematically higher than the those for the EOM-CCS. Thus the inclusion of the NL term in the cumulant has the effect of transferring intensity from the satellites back into the quasiparticle peak.

\section{Satellite properties}

The spectral function $A_{c}(\omega)$, which characterizes the excitation spectrum for a given level, is defined as

$$
A_{c}(\omega)=-\frac{1}{\pi} \operatorname{Im} G_{c}(\omega) .
$$

This spectrum characterizes the intrinsic, inelastic losses in the system as measured, e.g., in XPS. Fig. 1 shows a comparison of $A_{c}(\omega)$ for the $10 e$ systems computed with the aug-cc-pVDZ basis set and the full NL cumulant form, as a function of EOM-CCS approximation. Similar figures for the other basis sets and for the linear approximation to the cumulant are given in the SI. To make the comparison between different systems more clear, the spectral functions in Fig. 1 are plotted with respect to the bare core-hole energy $\epsilon_{c}$ (which corresponds

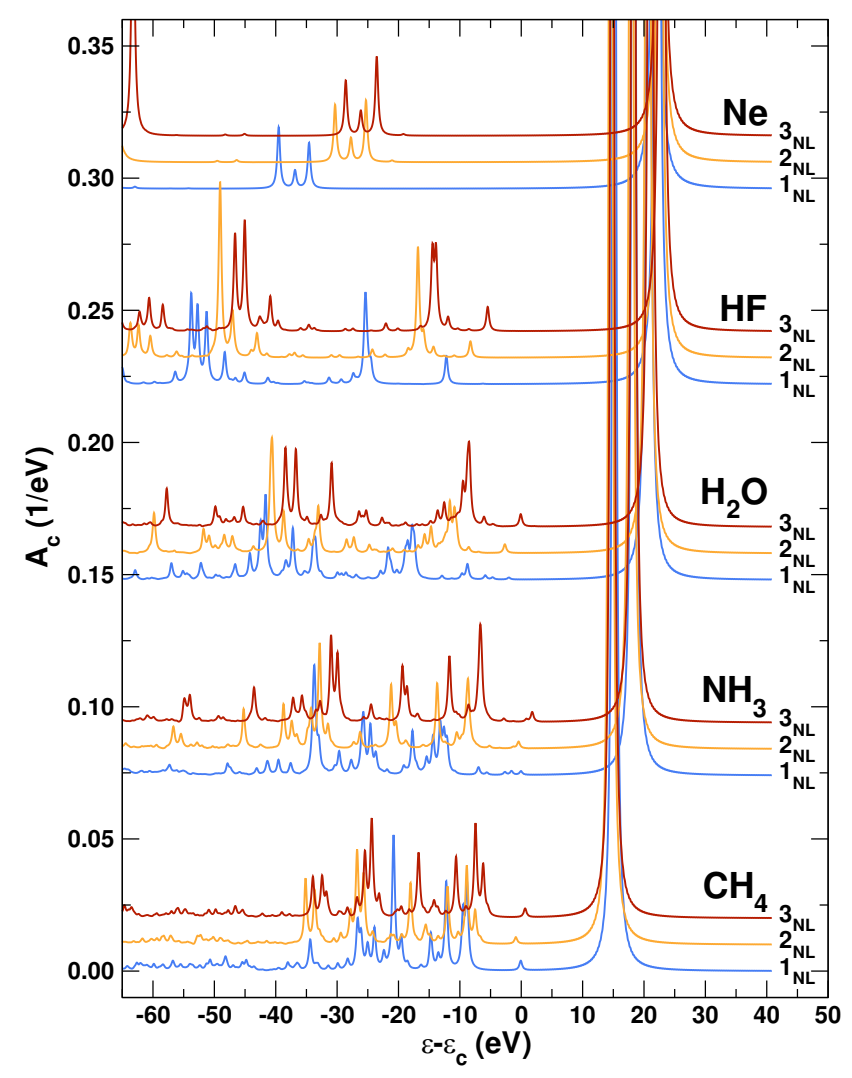

FIG. 1: Core spectral function $A_{c}$ of the $10 e$ systems computed with the aug-cc-pVDZ basis set and the full NL cumulant form, as a function of EOM-CCS approximation $1_{N L}$ (blue), $2_{N L}$ (orange), and $3_{N L}$ (red).

to $E_{K T}^{B}=-\epsilon_{c}$, where $E_{K T}^{B}$ is the Koopmans' Theorem binding energy in Table I). With this reference, the position of the quasiparticle peak corresponds to the relaxation energy

$$
\Delta=\epsilon_{B}-\left|\epsilon_{c}\right|=\int d \omega \frac{\beta(\omega)}{\omega},
$$

as discussed in our original treatment. ${ }^{7}$ Given that the relaxation is inversely proportional to the mean core- 
TABLE II: Comparison of the quasiparticle strengths obtained with the aug-cc-pVDZ basis set, using the L and NL approximations to the cumulant and the 1-3 approximations of the EOM-CCS method. Note that the non-linear terms in the cumulant significantly increase the quasiparticle strength, while the 2nd-order Green's function approximation is even larger. The quadratic (2) and cubic terms (3) in the EOM have only minor effects.

\begin{tabular}{lrcccccccc}
\hline \hline System & DSE2 & GFCCSD & GFCC-i(2,3) & $\mathbf{1}_{L}$ & $\mathbf{2}_{L}$ & $\mathbf{3}_{L}$ & $\mathbf{1}_{N L}$ & $\mathbf{2}_{N L}$ & $\mathbf{3}_{N L}$ \\
\hline $\mathrm{CH}_{4}$ & 0.80 & 0.80 & 0.76 & 0.59 & 0.63 & 0.60 & 0.70 & 0.72 & 0.70 \\
$\mathrm{NH}_{3}$ & 0.77 & 0.81 & 0.75 & 0.60 & 0.62 & 0.59 & 0.71 & 0.72 & 0.70 \\
$\mathrm{H}_{2} \mathrm{O}$ & 0.76 & 0.82 & 0.77 & 0.63 & 0.63 & 0.60 & 0.73 & 0.73 & 0.71 \\
$\mathrm{HF}$ & 0.77 & 0.84 & 0.78 & 0.69 & 0.67 & 0.65 & 0.76 & 0.75 & 0.74 \\
$\mathrm{Ne}$ & 0.80 & 0.87 & 0.81 & 0.76 & 0.73 & 0.72 & 0.81 & 0.79 & 0.78 \\
\hline \hline
\end{tabular}

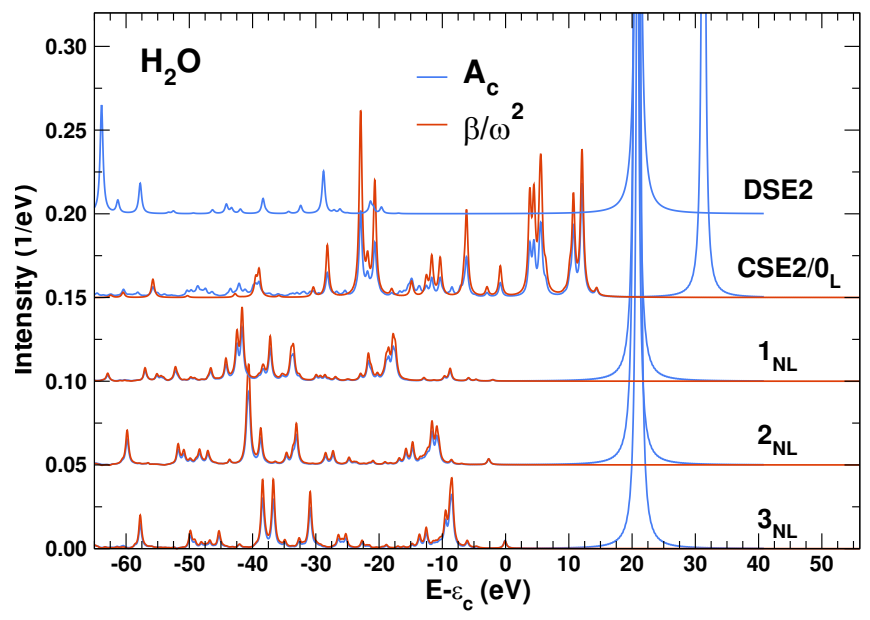

FIG. 2: Comparison of the core spectral function $A_{c}$ (blue) and the cumulant kernel $\beta(\omega) / \omega^{2}$ (red) for $\mathrm{H}_{2} \mathrm{O}$, computed with the aug-cc-pVDZ basis set and the full NL cumulant form, as a function of approximation $\left(1_{N L}-3_{N L}\right)$ of the EOMCCS method. For comparison we also include results obtained with the DSE2 and CSE2 methods, the latter being equivalent to the $0_{L}$ approximation.

valence interaction potential, which for these systems decreases from $\mathrm{CH}_{4}$ to $\mathrm{Ne}$, we see a blue shift trend in the quasiparticle position.

Fig. 1 also shows the satellite region near the quasiparticle peak. We find that the main effect of EOM-CCS approximation including non-linear corrections is a narrowing of the quasiparticle-satellite gap improving the agreement with experiment. Given that the quasiparticle position is nearly constant with respect to the level of approximation, most of the gap closing arises from an increase in the satellite energies. For most of the studied systems, we find that the satellite structure obtained with the quadratic approximation $\left(2_{N L}\right)$ is similar to that for the full method $\left(3_{N L}\right)$ up to the overall shift (see SI Fig. 6). The linear approximation shows a similar satellite weight distribution, but with some differences in the position of the particular features.

Fig. 2 shows a comparison between the cumulant kernel $\beta(\omega) / \omega^{2}$ and the satellite region of $A_{c}(\omega)$ for $\mathrm{H}_{2} \mathrm{O}$. As expected, the peaks in $\beta(\omega)$ for the EOM-CC cumulant correspond well to the inelastic losses in $A_{c}(\omega)$. Note however, that neither the DSE2 nor the CSE2 approximations are adequate to describe the satellite spectra of small molecules. The CSE2 significantly overestimates the quasiparticle relaxation energy, while the DSE2 gives a poor representation of the spectral function. As discussed in Sec. II C, given that the CSE2 and the minimal $\left(0_{L}\right)$ approximation to the EOM-CC method are equivalent, this error is corrected by the remaining terms in the EOM-CCS equations which bring the quasiparticle into good agreement with the DSE2 results and with experiment.

Finally, Fig. 3 shows a comparison of the full $\left(3_{N L}\right)$ EOM-CCS spectral functions obtained with the aug-ccpVDZ basis set, to available experimental XPS results. We focus mainly on the satellite region, and the EOMCCS results include scissors corrections to facilitate the comparison to experiment. We find that the agreement with experiment is quite satisfactory considering the simplicity of the EOM-CCS approximation. The satellite peaks arise from shake-up excitations, in which the creation of the core hole is accompanied by valence-valence excitations, i.e., density fluctuations of opposite sign. The need to include scissors corrections indicates that the $e^{T_{1}}$ approximation is not sufficient to fully account for the relaxation of the satellite transitions due to electronelectron interactions and dynamic screening of the corehole. However, the reasonable agreement obtained using a single scissors shift for each system points to missing dynamic correlation effects that would likely be accounted for with the inclusion of higher order cluster operators. For example, comparing GFCCSD and GFCC-i $(2,3)$ results, we did find that the satellite positions shift significantly towards the main quasiparticle peaks. For $\mathrm{H}_{2} \mathrm{O}$, the shift brought by the triples can be as large as $\sim 20 \mathrm{eV}$. However, the inclusion of higher order terms in the time domain in an effective and economic way is still an open question and is one of the future directions of research.

\section{CONCLUSIONS}

We have investigated the cumulant representation of the one-particle Green's function based on a real-time EOM-CC approximation. The logarithmic-derivative be- 


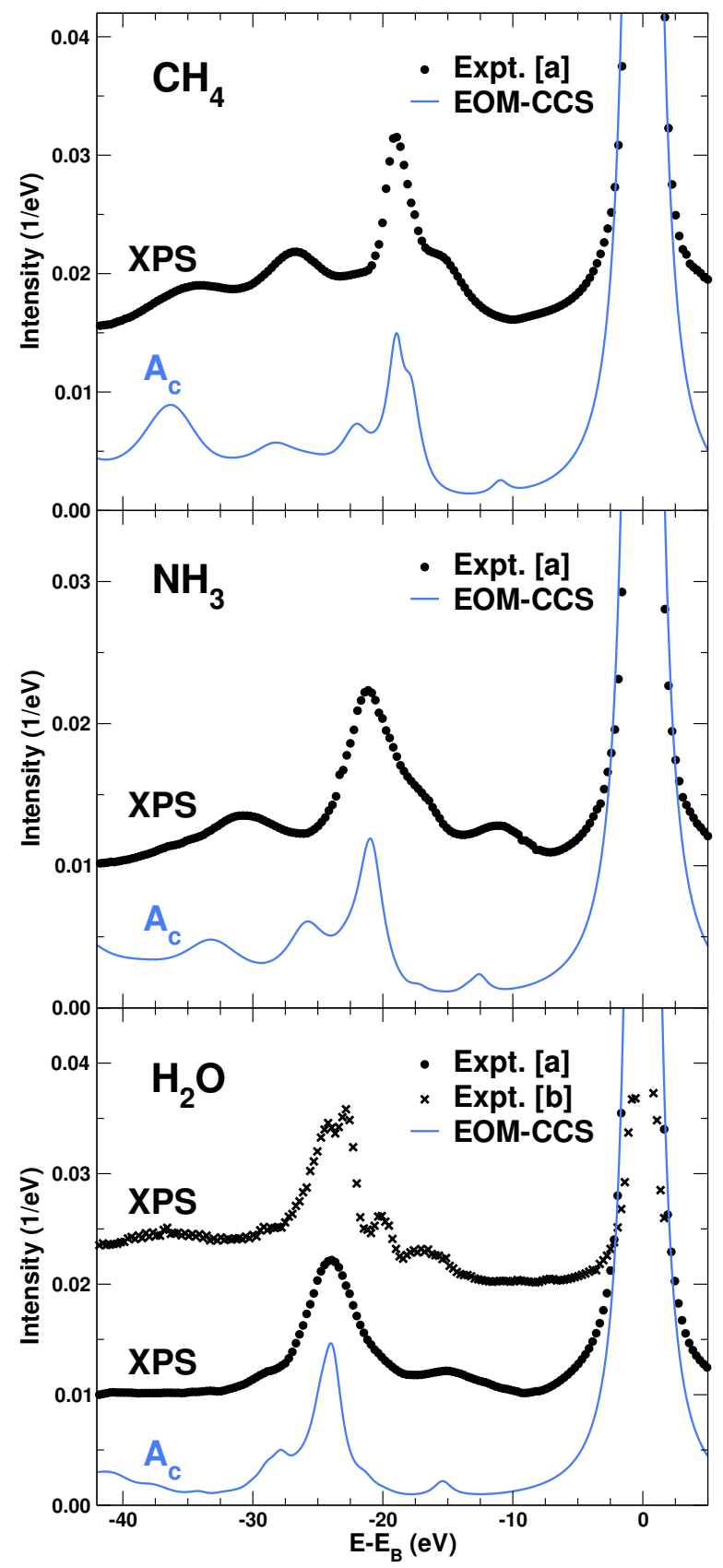

FIG. 3: Comparison between the experimental XPS (Expt.: [a] Ref. 28, [b] Ref. 29) and the aug-cc-pVDZ (3NL) EOMCCS core spectral functions of $\mathrm{CH}_{4}$ (top), $\mathrm{NH}_{3}$ (middle), and $\mathrm{H}_{2} \mathrm{O}$ (bottom) as a function of energy. The EOM-CCS results include scissors corrections of 5.7, 3.9 and $3.2 \mathrm{eV}$, respectively, to close the QP-satellite gap, and have been broadened roughly to match experiment.

havior of the EOM-CC yields an explicit exponential cumulant representation of the GF, with a non-perturbative expression for the cumulant in terms of the solutions to a set of coupled, first-order non-linear differential equations. Within the CC-singles approximation implemented here, the non-linear terms enter the formulation in two ways. First the expression for the cumulant is quadratic in the CC amplitudes $t_{i a}(t)$. Second, the EOM for the $\mathrm{CC}$ amplitudes have non-linear terms up to third order. To lowest order, i.e., 2nd-order in the electron-electron interaction, the retarded cumulant reduces to that defined by the 2nd-order self energy (SE2). We also found that the time-kernel in the EOM-CC is directly analogous to that in the static CC equations for the ground state, thus simplifying the implementation of our approach in current codes. As a quantitative test, we have applied the EOM-CC cumulant approach to the core-hole Green's function for a number of small molecular systems. We find that the EOM-CC cumulant approach with only CC-singles excitations yields accurate quasiparticle properties such as core-level binding energies, as well as an approximate treatment of satellite shapes. The observed improvement over other approaches likely stems from the implicit excitations present in the exponential form of the cumulant ansatz. Although the 2nd-order approximation gives reasonable results for the quasiparticle energy with the Dyson equation, the 2nd-order cumulant approximation for the relaxation energy has substantial errors, and the spectral function is poorly described. This behavior is in contrast to that observed in condensed matter, where the $G W+C$ approach $^{5,30,31}$ based on a cumulant from the 2nd-order GW self-energy and the quasi-boson approximation has proved to be advantageous for a description of satellites in the spectral function. ${ }^{2,5}$ Physically, this difference appears to reflect the particle-hole character of excitations in molecular systems, which leads to a sparser satellite structure without multiple-bosonic excitations. ${ }^{15}$ This suggests that the inclusion of nonlinear corrections to the cumulant is generally important for understanding the nature of electronic excitations in molecular systems.

Data Availability Statement: The data that support the findings of this study are available from the corresponding author upon reasonable request.

\section{Acknowledgments}

This work was supported by the Computational Chemical Sciences Program of the U.S. Department of Energy, Office of Science, BES, Chemical Sciences, Geosciences and Biosciences Division in the Center for Scalable and Predictive methods for Excitations and Correlated phenomena (SPEC) at PNNL. 
${ }^{2}$ L. Hedin, J. Phys.: Condens. Matter 11, R489 (1999).

3 B. Peng and K. Kowalski, Phys. Rev. A 94, 062512 (2016).

4 B. Peng and K. Kowalski, J. Chem. Theory Comput. 14, 4335 (2018).

5 J. Zhou, J. Kas, L. Sponza, I. Reshetnyak, M. Guzzo, C. Giorgetti, M. Gatti, F. Sottile, J. Rehr, and L. Reining, J. Chem. Phys. 143, 184109 (2015).

${ }^{6}$ D. C. Langreth, Phys. Rev. B 1, 471 (1970).

7 J. Rehr, F. Vila, J. Kas, N. Hirshberg, K. Kowalski, and B. Peng, J. Chem. Phys. 152, 174113 (2020).

8 F. Aryasetiawan, L. Hedin, and K. Karlsson, Phys. Rev. Lett. 77, 2268 (1996).

9 J. J. Kas, J. J. Rehr, and L. Reining, Phys. Rev. B 90, $085112(2014)$.

10 J. Linderberg and Y. Öhrn, Propagators in Quantum Chemistry, Theoretical chemistry (Wiley, 2004), ISBN 9780471662570 .

11 A. Szabo and N. Ostlund, Modern Quantum Chemistry: Introduction to Advanced Electronic Structure Theory, Dover Books on Chemistry (Dover Publications, 1996), ISBN 9780486691862.

12 T. D. Crawford and H. F. Schaefer, Reviews in computational chemistry 14, 33 (2000).

13 B. Peng and K. Kowalski, Phys. Rev. A 94, 062512 (2016).

14 P. Nozieres and C. D. Dominicis, Phys. Rev. 178, 1097 (1969).

15 M. Tzavala, J. J. Kas, L. Reining, and J. J. Rehr, Nonlinear response in the cumulant expansion for core hole photoemission (2020), arXiv:2005.01857.

16 in NIST Computational Chemistry Comparison and Benchmark Database, edited by R. D. Johnson III (NIST, 2019), vol. Release 20 of NIST Standard Reference Database Number 101, URL http://cccbdb.nist.gov.

17 R. Muller (2004), URL http://pyquante.sourceforge. net.

18 T. H. Dunning, The Journal of Chemical Physics 90, 1007 (1989).

19 R. A. Kendall, T. H. Dunning, and R. J. Harrison, J. Chem. Phys. 96, 6796 (1992).

20 A. Quarteroni, R. Sacco, and F. Saleri, Numerical Mathematics (Springer-Verlag Berlin Heidelberg, 2007).

21 B. Peng and K. Kowalski, The Journal of Chemical Physics 149, 214102 (2018).

22 B. Peng, R. Van Beeumen, D. B. Williams-Young, K. Kowals ki, and C. Yang, Journal of Chemical Theory and Computation 15, 3185 (2019).

${ }^{23}$ T. Karlsen, K. J. Brve, L. J. Sthre, K. Wiesner, M. Bssler, and S. Svensson, Journal of the American Chemical Society 124, 7866 (2002).

${ }^{24}$ T. Buttersack, P. E. Mason, R. S. McMullen, T. Martinek, K. Brezina, D. Hein, H. Ali, C. Kolbeck, C. Schewe, S. Malerz, et al., Journal of the American Chemical Society 141, 1838 (2019).

${ }^{25}$ F. Vies, C. Sousa, and F. Illas, Phys. Chem. Chem. Phys. 20, 8403 (2018).

26 Atomic Data and Nuclear Data Tables 31, 433 (1984), ISSN 0092-640X.

27 G. P. Williams, X-Ray Data Booklet: Section 1.1 ELECTRON BINDING ENERGIES (Lawrence Berkeley National Laboratory, Berkeley, 2009).

28 D. K. Creber, J. S. Tse, and G. M. Bancroft, The Journal of Chemical Physics 72, 4291 (1980).

${ }^{29}$ Chemical Physics Letters 422, 51 (2006), ISSN 0009-2614.
30 J. Lischner, D. Vigil-Fowler, and S. G. Louie, Phys. Rev. Lett. 110, 146801 (2013).

31 B. Gumhalter, V. Kovač, F. Caruso, H. Lambert, and F. Giustino, Phys. Rev. B 94, 035103 (2016). 


\title{
Supplementary Information: Real-time coupled-cluster approach for the cumulant Green's function
}

\author{
F. D. Vila, J. J. Rehr, and J. J. Kas \\ Department of Physics, University of Washington, Seattle, WA 98195 \\ K. Kowalski \\ William R. Wiley Environmental Molecular Sciences Laboratory, \\ Battelle, Pacific Northwest National Laboratory, \\ K8-91, P. O. Box 999, Richland, Washington 99352 \\ B. Peng \\ Physical Sciences Division, Pacific Northwest National Laboratory, Richland, WA 99354
}

(Dated: June 16, 2020)

\begin{abstract}
This Supplementary Information presents results that are too detailed for the main manuscript, including more detailed discussion of the theory derivations in the main manuscript, computational details, results for other basis sets, and more results that are too technical for the main manuscript.
\end{abstract}




\section{THEORY}

\section{A. Retarded Green's function in spin-orbital basis}

The real-space, real-time retarded Green's function is defined as

$$
\begin{aligned}
G\left(x, x^{\prime}\right)= & G\left(r t, r^{\prime} t^{\prime}\right)= \\
& -i \Theta\left(t-t^{\prime}\right)\left\langle 0\left|\left\{\psi(r t), \psi^{\dagger}\left(r^{\prime} t^{\prime}\right)\right\}\right| 0\right\rangle
\end{aligned}
$$

where $|0\rangle$ is the ground state of the system, $\psi^{\dagger}\left(r^{\prime} t^{\prime}\right)$ and $\psi(r t)$ are, respectively, the creation and annhilation field operators at $r^{\prime} t^{\prime}$ and $r t$, and $\{$,$\} indicates the anticonmutation operator.$ By introducing a basis set of single-particle spin-orbitals $\left\{\phi_{p}(r)\right\}$, we can express any twopositions, two-times operator $M$ as:

$$
M\left(r t, r^{\prime} t^{\prime}\right)=\sum_{p q} \phi_{p}^{*}(r) M_{p q}\left(t, t^{\prime}\right) \phi_{q}\left(r^{\prime}\right)
$$

where the matrix elements are defined as:

$$
M_{p q}\left(t, t^{\prime}\right)=\int d r d r^{\prime} \phi_{p}^{*}(r) M\left(r t, r^{\prime} t^{\prime}\right) \phi_{q}\left(r^{\prime}\right) .
$$

Inserting these definitions into Eq. (1) we obtain the Green's function matrix expressed in the spin-orbital basis $\left\{\phi_{p}(r)\right\}$ :

$$
G_{p q}\left(t, t^{\prime}\right)=-i \Theta\left(t-t^{\prime}\right)\left\langle 0\left|\left\{a_{p}(t), a_{q}^{\dagger}\left(t^{\prime}\right)\right\}\right| 0\right\rangle,
$$

where the creation and annhilation operators $a_{p}^{\dagger}(t)$ and $a_{q}\left(t^{\prime}\right)$ are associated with the spinorbitals $\phi_{p}$ and $\phi_{q}$ respectively.

B. From the real-space time-domain Dyson equation to the cumulant ansatz in a spin-orbital basis

The real-space, time-domain form of the Dyson equation

$$
\begin{aligned}
G\left(x, x^{\prime}\right)= & G^{0}\left(x, x^{\prime}\right)+ \\
& \int d x_{1} d x_{2} G^{0}\left(x, x_{1}\right) \Sigma\left(x_{1}, x_{2}\right) G\left(x_{2}, x^{\prime}\right)
\end{aligned}
$$

can also be cast in matrix form (after including time translation invariance):

$$
\hat{G}(t)=\hat{G}^{0}(t)+\int d t_{1} d t_{2} \hat{G}^{0}\left(t-t_{1}\right) \hat{\Sigma}\left(t_{1}-t_{2}\right) \hat{G}\left(t_{2}\right) .
$$


Expanding the matrix form of the cumulant ansatz

$$
\hat{G}(t)=\hat{G}^{0}(t) e^{\hat{C}(t)}
$$

and Eq. (6) to first order

$$
\begin{aligned}
& \hat{G}(t)=\hat{G}^{0}(t)+\hat{G}^{0}(t) \hat{C}(t)+\ldots \\
& \hat{G}(t)=\hat{G}^{0}(t)+\int d t_{1} d t_{2} \hat{G}^{0}\left(t-t_{1}\right) \hat{\Sigma}\left(t_{1}-t_{2}\right) \hat{G}^{0}\left(t_{2}\right)+\ldots
\end{aligned}
$$

and equating the second terms in the right hand sides we get:

$$
\hat{G}^{0}(t) \hat{C}(t)=\int d t_{1} d t_{2} \hat{G}^{0}\left(t-t_{1}\right) \hat{\Sigma}\left(t_{1}-t_{2}\right) \hat{G}^{0}\left(t_{2}\right)
$$

For a typical matrix element we obtain

$$
\sum_{r} G_{p r}^{0}(t) C_{r q}(t)=\sum_{r s} \int d t_{1} d t_{2} G_{p r}^{0}\left(t-t_{1}\right) \Sigma_{r s}\left(t_{1}-t_{2}\right) G_{s q}^{0}\left(t_{2}\right),
$$

Shifting the $\omega$ integration variable and using the Fourier transform of the cumulant we have:

$$
C_{p q}(\omega)=i G_{p p}^{0}\left(\omega+\epsilon_{p}\right) \Sigma_{p q}\left(\omega+\epsilon_{p}\right) G_{q q}^{0}\left(\omega+\epsilon_{p}\right) .
$$

Finally, introducing the frequency domain form of $G_{p p}^{0}=\left(\omega-\epsilon_{p}+i \delta\right)^{-1}$ :

$$
C_{p q}(\omega)=\frac{i \Sigma_{p q}\left(\omega+\epsilon_{p}\right)}{(\omega+i \delta)\left(\omega+\epsilon_{p}-\epsilon_{q}+i \delta\right)} .
$$

If we approximate $\Sigma_{p q}(\omega) \simeq \Sigma_{p p}(\omega) \delta_{p q}$, then

$$
C_{p p}(\omega)=\frac{i \Sigma_{p p}\left(\omega+\epsilon_{p}\right)}{(\omega+i \delta)^{2}}
$$

or, in the time domain

$$
C_{p p}(t)=\int \frac{d \omega}{2 \pi} \frac{i \Sigma_{p p}\left(\omega+\epsilon_{p}\right)}{(\omega+i \delta)^{2}} e^{-i \omega t} .
$$

We can now return to the time domain, inserting into Eq. (7) and taking into account that the matrix exponential is now diagonal:

$$
G_{p p}(t)=i \Theta(t) e^{-i \epsilon_{p} t+C_{p p}(t)}
$$

which is the standard form of the diagonal cumulant. 


\section{Coupled Cluster Green's function in time}

In this section we derive a compact form for the full Coupled Cluster Green's function that can be used for further derivation of time-domain approximations. Starting with Eq. (17) in Ref. 1 (from now on referred as "PK") we have

$$
G_{p q}^{R}(\omega)=\left\langle\Phi\left|(1+\Lambda) e^{-T} a_{q}^{\dagger}\left(\omega+\left(H-E_{0}\right)-i \delta\right)^{-1} a_{p} e^{T}\right| \Phi\right\rangle
$$

and inserting the $I=e^{-T} e^{T}$ we get

$$
G_{p q}^{R}(\omega)=\left\langle\Phi\left|(1+\Lambda) \bar{a}_{q}^{\dagger}\left(\omega+\bar{H}_{N}\right)^{-1} \bar{a}_{p}\right| \Phi\right\rangle
$$

where $\bar{O}=e^{-T} O e^{T}$ is the similarity transformed form of the $O$ operator, $H_{N}$ is the normal ordered hamiltonian, and using the Baker-Campbell-Hausdorff (BCH) relation we have that

$$
\begin{aligned}
& \bar{a}_{p}=a_{p}+\left[a_{p}, T\right], \\
& \overline{a_{q}^{\dagger}}=a_{q}^{\dagger}+\left[a_{q}^{\dagger}, T\right] .
\end{aligned}
$$

For simplicity, we now make the convergence factor $-i \delta$ implicit in the energy $\omega$.

Following PK, we introduce the $X_{p}(\omega), Z_{q}(\omega)$, and $W_{q}(\omega)$ operators, which are solutions to the following equations:

$$
\begin{gathered}
X_{p}(\omega)|\Phi\rangle=\left(\omega+\bar{H}_{N}\right)^{-1} \bar{a}_{p}|\Phi\rangle, \\
\langle\Phi| Z_{q}(\omega)=\langle\Phi|(1+\Lambda) \bar{a}_{q}^{\dagger}\left(\omega+\bar{H}_{N}\right)^{-1},
\end{gathered}
$$

and

$$
\langle\Phi|(1+\Lambda) W_{q}(\omega)=\langle\Phi|(1+\Lambda) \bar{a}_{q}^{\dagger}\left(\omega+\bar{H}_{N}\right)^{-1}
$$

These operators have the following expansions in the $N-1$ Fock space:

$$
\begin{aligned}
X_{p}(\omega)= & \sum_{i} x^{i}(\omega)_{p} a_{i}+ \\
& \frac{1}{2 !} \sum_{i j, a} x_{a}^{i j}(\omega)_{p} a_{a}^{\dagger} a_{j} a_{i}+\ldots \\
Z_{q}(\omega)= & \sum_{i} z_{i}(\omega)_{q} a_{i}^{\dagger}+ \\
& \frac{1}{2 !} \sum_{i j, a} z_{i j}^{a}(\omega)_{p} a_{i}^{\dagger} a_{j}^{\dagger} a_{a}+\ldots
\end{aligned}
$$


and

$$
\begin{aligned}
W_{q}(\omega)= & \sum_{i} w_{i}(\omega)_{q} a_{i}^{\dagger}+ \\
& \frac{1}{2 !} \sum_{i j, a} w_{i j}^{a}(\omega)_{p} a_{i}^{\dagger} a_{j}^{\dagger} a_{a}+\ldots
\end{aligned}
$$

Eqs. (17)-(26) are a summary of the formulation in PK. Now, using

$$
\operatorname{IFT}\left[\left(\omega+\bar{H}_{N}-i \delta\right)^{-1}\right]=i \Theta(-t) e^{i \bar{H}_{N} t}
$$

where IFT is the inverse Fourier transform, we can write $G_{p q}^{R}(\omega)$ in the time-domain as:

$$
G_{p q}^{R}(t)=i\left\langle\Phi\left|(1+\Lambda) \bar{a}_{q}^{\dagger} e^{i \bar{H}_{N} t} \bar{a}_{p}\right| \Phi\right\rangle
$$

where from now on we assume that $t<0$ to remove all the $\Theta(-t)$ functions. We can also convert the equations defining the $X_{p}(\omega), Z_{q}(\omega)$, and $W_{q}(\omega)$ operators as:

$$
\begin{gathered}
X_{p}(t)|\Phi\rangle=i e^{i \bar{H}_{N} t} \bar{a}_{p}|\Phi\rangle \\
\langle\Phi| Z_{q}(t)=i\langle\Phi|(1+\Lambda) \bar{a}_{q}^{\dagger} e^{i \bar{H}_{N} t}
\end{gathered}
$$

and

$$
\langle\Phi|(1+\Lambda) W_{q}(t)=i\langle\Phi|(1+\Lambda) \overline{a_{q}^{\dagger}} e^{i \bar{H}_{N} t}
$$

where the $X_{p}(t), Z_{q}(t)$, and $W_{q}(t)$ are defined simply by the inverse Fourier transform of their coefficients in their excitation expansions (Eqs. (24)-(26)).

Based on the properties of the $\bar{H}_{N}$, i.e., where one assumes that the CC equations $Q \bar{H}_{N}|\Phi\rangle=0$ are satisfied, where $Q$ denotes the projection operator onto the space spanned by excitations with respect to $|\Phi\rangle$ Slater determinants, we can prove that, in full analogy with the frequency representation, the $X_{p}(t)$ operator can be expressed in terms of connected diagrams only. To this end we expand $X_{p}(t)$ in term of powers of the $\bar{H}_{N}$ operator

$$
\begin{aligned}
X_{p}(t)|\Phi\rangle & =i e^{i \bar{H}_{N} t} \overline{a_{p}}|\Phi\rangle, \\
& =i\left\{\sum_{n=0}^{\infty} \frac{1}{n !}(i)^{n} t^{n}\left(\bar{H}_{N}\right)^{n} \overline{a_{p}}\right\}|\Phi\rangle
\end{aligned}
$$

Now we assume that we are dealing with the exact CC theory. This assumption plays a crucial role in proving the connected character of $X_{p}(t)$ since all approximate approaches 

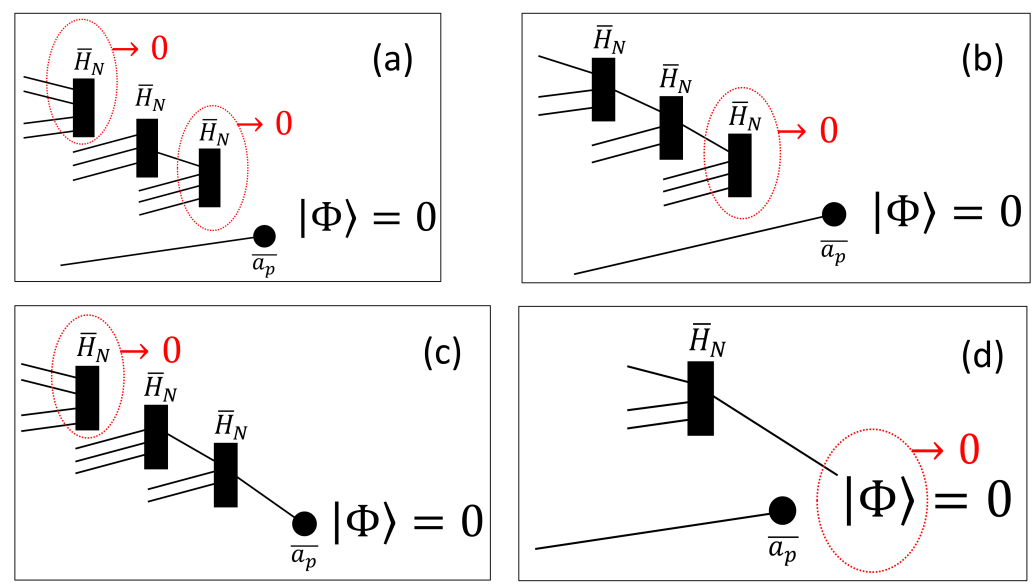

FIG. 1: Typical examples of diagrams not contributing to the general term in Eq. (34).

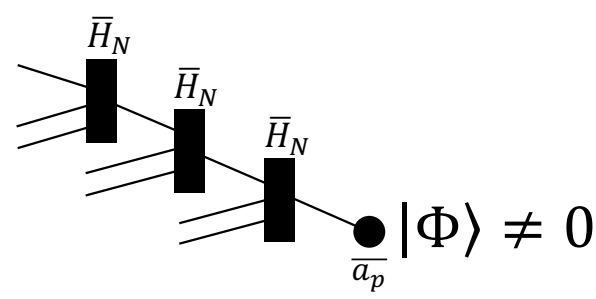

FIG. 2: Example of a connected diagram that contributes to the general term in Eq. (34).

can be build using connected properties of the exact formulation. Using Wick's theorem for the particle-hole formalism to analize a general term in the expansion in Eq. (33):

$$
\left(\bar{H}_{N}\right)^{n} \overline{a_{p}}|\Phi\rangle
$$

we can identify several classes of diagrams contributing to $\left(\bar{H}_{N}\right)^{n} \overline{a_{p}}|\Phi\rangle$ (see Figs. (1) and (2)). It can be easily verified that all disconnected diagrams (typical examples of these are shown in Fig. (1a-1d)) disappear due to the existence of vertices representing projections of CC equations (i.e., $\bar{H}_{N}$ matrix elements with all particle-hole creation lines, that is, all "legs", located to the left of the corresponding matrix element or diagrammatic vertex, see Fig. (1a1c)) or due to the existence of the uncontracted particle-hole line (lines) that annihilates the reference function $|\Phi\rangle$ as shown in Fig. (1d). Consequently, the only diagrams contributing to $X_{p}(t)$ are connected diagrams (Fig. $\left.(2)\right)$ which can be symbolically denoted as

$$
X_{p}(t)|\Phi\rangle=i\left\{e^{i \bar{H}_{N} t} \overline{a_{p}}\right\}_{C}|\Phi\rangle
$$

where the subscript "C" designates connected part of a given operator expression. 
Using the equations above for $X_{p}(t), Z_{q}\left(0^{-}\right)$, and $W_{q}\left(0^{-}\right)$, we can re-write $G_{p q}^{R}(t)$ as:

$$
\begin{aligned}
G_{p q}^{R}(t) & =\left\langle\Phi\left|(1+\Lambda) \overline{a_{q}^{\dagger}} X_{p}(t)\right| \Phi\right\rangle \\
& =-i\left\langle\Phi\left|Z_{q}\left(0^{-}\right) X_{p}(t)\right| \Phi\right\rangle \\
& =-i\left\langle\Phi\left|(1+\Lambda) W_{q}\left(0^{-}\right) X_{p}(t)\right| \Phi\right\rangle
\end{aligned}
$$

The first equality is just the time-domain version of Eq. (27) in PK. Note that the $Z_{q}$ and $W_{q}$ operators are computed as the limit $t \rightarrow 0^{-}$to avoid any issues with the poorly defined nature of $\Theta(-t)$ at 0 .

We now proceed to expand the second equality in Eq. (36). We choose to expand this form of $G_{p q}^{R}(t)$ because it does not include the $\Lambda$ terms (which are implicit in the definition of $\left.Z_{q}\left(0^{-}\right)\right)$and is thus simpler. Inserting the time dependent definitions of $X_{p}(t)$ and $Z_{q}\left(0^{-}\right)$ into $G_{p q}^{R}(t)$ we have

$$
\begin{aligned}
i G_{p q}^{R}(t)=\langle\Phi|\left(\sum_{i} z_{i}\left(0^{-}\right)_{q}\left\{a_{i}^{\dagger}\right\}+\right. & \left.\frac{1}{2 !} \sum_{i j, a} z_{i j}^{a}\left(0^{-}\right)_{p}\left\{a_{i}^{\dagger} a_{j}^{\dagger} a_{a}\right\}+\ldots\right) \\
& \left(\sum_{k} x^{k}(t)_{p}\left\{a_{k}\right\}+\frac{1}{2 !} \sum_{k l, b} x_{b}^{k l}(t)_{p}\left\{a_{b}^{\dagger} a_{l} a_{k}\right\}+\ldots\right)|\Phi\rangle
\end{aligned}
$$

where, to simplify the rest of the calculations, we also introduced the normal ordered forms of the operators, indicated using Bartlett's style notation $\{\ldots\}$ rather than the PK one with $N[\ldots]$. Before going any further it is helpful to analyze the general form of a generic matrix element for the different products:

$$
\left\langle\Phi\left|\{\underbrace{a_{i}^{\dagger} a_{j}^{\dagger} \ldots a_{b} a_{a}}_{n \text { ops }}\}\{\underbrace{a_{c}^{\dagger} a_{d}^{\dagger} \ldots a_{l} a_{k}}_{m \text { ops }}\}\right| \Phi\right\rangle=\sum_{\text {FC }}\left\langle\Phi\left|\left\{a_{i}^{\dagger} a_{j}^{\dagger} \ldots a_{b} a_{b} a_{a} a_{c}^{\dagger} a_{d}^{\dagger} \ldots a_{l} a_{k}\right\}\right| \Phi\right\rangle
$$

where, according to the generalized Wick's theorem (GWT), we compute the Fermi vacuum expectation value of the product of two normal ordered operator sets by summing over all possible full contractions (FC). However, for $n \neq m$, the are no possible full contractions between the two operator sets, thus, the "cross" terms in Eq. (37) are all zero. For example, we are only left with terms of the form:

$$
\left\langle\Phi\left|\left\{a_{i}^{\dagger}\right\}\left\{a_{k}\right\}\right| \Phi\right\rangle=\left\langle\Phi\left|\left\{\vec{a}_{i}^{\dagger} a_{k}\right\}\right| \Phi\right\rangle=\delta_{i k}
$$

for 0 excitation order (i.e. order 1 in $\mathrm{PK}$ ),

$$
\begin{array}{r}
\left\langle\Phi\left|\left\{a_{i}^{\dagger} a_{j}^{\dagger} a_{a}\right\}\left\{a_{b}^{\dagger} a_{l} a_{k}\right\}\right| \Phi\right\rangle=\left\langle\Phi\left|\left\{a_{i}^{\dagger} a_{j}^{\dagger} a_{a} a_{b}^{\dagger} a_{l} a_{k}\right\}\right| \Phi\right\rangle+\left\langle\Phi\left|\left\{a_{i}^{\dagger} a_{j}^{\dagger} a_{a} a_{b}^{\dagger} a_{l} a_{k}\right\}\right| \Phi\right\rangle= \\
-\delta_{i l} \delta_{j k} \delta_{a b}+\delta_{i k} \delta_{j k} \delta_{a b}
\end{array}
$$


for 1 excitation order (i.e. order 2 in PK), etc. The pattern is fairly clear: each order produces $h ! \times p$ !, where $h$ and $p$ are the number of hole and particle operators in the products. Thus PK order 1 produces one term, order 2 produces 2 , order 3 would produce 12, etc. Thus we can write:

$$
i G_{p q}^{R}(t)=\sum_{i} z_{i}\left(0^{-}\right)_{q} x^{i}(t)_{p}+\frac{1}{4} \sum_{i j, a} z_{i j}^{a}\left(0^{-}\right)_{p} x_{a}^{i j}(t)_{p}-\frac{1}{4} \sum_{i j, a} z_{i j}^{a}\left(0^{-}\right)_{p} x_{a}^{j i}(t)_{p}+\ldots
$$

This sum can be generalized by recognizing that the $x_{a}^{i j}(t)_{p}$ coefficients are antisymmetric with respect to index swaps, and that $x_{a}^{i i}(t)_{p}=0$, thus

$$
\begin{aligned}
i G_{p q}^{R}(t)= & \\
& \sum_{i} z_{i}\left(0^{-}\right)_{q} x^{i}(t)_{p}+ \\
+ & \frac{1}{2} \sum_{i j, a} z_{i j}^{a}\left(0^{-}\right)_{p} x_{a}^{i j}(t)_{p}+ \\
+ & \frac{1}{6} \sum_{i j k, a b} z_{i j k}^{a b}\left(0^{-}\right)_{p} x_{a b}^{i j k}(t)_{p}+\ldots
\end{aligned}
$$

\section{Perturbation theory of $G_{p q}^{R}(t)$}

In this section we demonstrate that the full form of the Coupled Cluster Green's function can be reduced to a cumulant form when approximated as a perturbations series. Although this analysis is valid for any CC level (CCD, CCSD, CCSDT, etc.) with HF orbitals and Moller Plesset MBPT, here for simplicity we limit the CC expansion to doubles $\left(T=T_{2}=\right.$

$\left.\frac{1}{4} \sum_{i j a b} t_{i j}^{a b} a_{a}^{\dagger} a_{b}^{\dagger} a_{j} a_{i}, \Lambda=\Lambda_{2}=\frac{1}{4} \sum_{i j a b} \lambda_{i j}^{a b} a_{i}^{\dagger} a_{j}^{\dagger} a_{b} a_{a}\right)$ and expand the $\bar{a}_{p}$ and $\overline{a_{q}^{\dagger}}$ operators into their connected forms:

$$
\begin{aligned}
G_{p q}^{R}(\omega) & = \\
& \left\langle\Phi\left|\left(1+\Lambda_{2}\right)\left(a_{q}^{\dagger}+\left(a_{q}^{\dagger} T_{2}\right)_{C}\right) X_{p}(\omega)\right| \Phi\right\rangle+ \\
& \left\langle\Phi\left|\left(1+\Lambda_{2}\right)\left(a_{p}+\left(a_{p} T_{2}\right)_{C}\right) Y_{q}(\omega)\right| \Phi\right\rangle
\end{aligned}
$$

where the $X_{p}$ and $Y_{q}$ operators are

$$
\begin{aligned}
& X_{p}(\omega)|\Phi\rangle=\left(\omega+\bar{H}_{N}+i \delta\right)^{-1} \bar{a}_{p}|\Phi\rangle, \\
& Y_{q}(\omega)|\Phi\rangle=\left(\omega-\bar{H}_{N}+i \delta\right)^{-1} \bar{a}_{q}^{\dagger}|\Phi\rangle,
\end{aligned}
$$


with components

$$
\begin{aligned}
& X_{p}(\omega)=\sum_{i} x^{i}(\omega)_{p} a_{i}+\frac{1}{2 !} \sum_{i j, a} x_{a}^{i j}(\omega)_{p} a_{a}^{\dagger} a_{j} a_{i}=X_{1, p}(\omega)+X_{2, p}(\omega), \\
& Y_{q}(\omega)=\sum_{a} y_{a}(\omega)_{q} a_{a}^{\dagger}+\frac{1}{2 !} \sum_{i, a b} y_{a b}^{i}(\omega)_{q} a_{a}^{\dagger} a_{b}^{\dagger} a_{i}=Y_{1, q}(\omega)+Y_{2, q}(\omega)
\end{aligned}
$$

We proceed in the frequency domain because this simplifies the perturbation analysis. Each term in Eq. (43) generates six possible terms, but, analyzing their corresponding diagrams we can see that there are only three non-zero terms when $p, q \in$ occ:

$$
G_{p q}^{R}(\omega)=\left\langle\Phi\left|a_{q}^{\dagger} X_{1, p}(\omega)\right| \Phi\right\rangle+\left\langle\Phi\left|\Lambda_{2}\left(a_{q}^{\dagger} T_{2}\right)_{C} X_{1, p}(\omega)\right| \Phi\right\rangle+\left\langle\Phi\left|\Lambda_{2} a_{p} Y_{2, q}(\omega)\right| \Phi\right\rangle .
$$

After computing each term we get:

$$
G_{p q}^{R}(\omega)=x^{q}(\omega)_{p}-\frac{1}{2} \sum_{i j a b} \lambda_{i j}^{a b} t_{a b}^{q j} x^{i}(\omega)_{p}-\frac{1}{2} \sum_{i a b} \lambda_{p i}^{a b} y_{a b}^{i}(\omega)_{q}
$$

This is the form of the Green's function which can be used to approximate $G_{p q}^{R}$ using perturbation theory. For this purpose we write each of the coefficients in Eq. (49) up to second order in a perturbation parameter $\lambda$, e.g. $t_{p q}^{r s}=t_{p q}^{(0) r s}+\lambda t_{p q}^{(1) r s}+\lambda^{2} t_{p q}^{(2) r s}$, etc. By keeping only terms up to second order we get:

$$
\begin{gathered}
G_{p q}^{(0) R}(\omega)=x^{(0) q}(\omega)_{p} \\
G_{p q}^{(1) R}(\omega)=x^{(1) q}(\omega)_{p}-\frac{1}{2} \sum_{i a b} \lambda_{p i}^{(1) a b} y_{a b}^{(0) i}(\omega)_{q} \\
G_{p q}^{(2) R}(\omega)=x^{(2) q}(\omega)_{p}-\frac{1}{2} \sum_{i j a b} \lambda_{i j}^{(1) a b} t_{a b}^{(1) q j} x^{(0) i}(\omega)_{p}-\frac{1}{2} \sum_{i a b}\left(\lambda_{p i}^{(1) a b} y_{a b}^{(1) i}(\omega)_{q}+\lambda_{p i}^{(2) a b} y_{a b}^{(0) i}(\omega)_{q}\right)
\end{gathered}
$$

It is easy to prove that $x^{(1) q}(\omega)_{p}=0$ and that $x^{(0) q}(\omega)_{p}=x^{(0) p}(\omega)_{p} \delta_{p q}$. It can also be easily proven that $Y_{q}^{(0)}(\omega)=0$, resulting in $G_{p q}^{(1) R}(\omega)=0$. After this, the retarded Green's function to second order is:

$$
G_{p q}^{R}(\omega)=x^{(0) p}(\omega)_{p} \delta_{p q}+x^{(2) q}(\omega)_{p}-\frac{1}{2} \sum_{i a b} \lambda_{p i}^{(1) a b}\left(t_{a b}^{(1) q i} x^{(0) p}(\omega)_{p}+y_{a b}^{(1) i}(\omega)_{q}\right)
$$

Here we skip the derivation of each of the coefficients in term of the two-particle integrals and HF eigenvalues and simply list expressions:

$$
x^{(0) p}(\omega)_{p}=\frac{1}{\left(\omega-\epsilon_{p}\right)}
$$




$$
\begin{gathered}
x^{(2) q}(\omega)_{p}=\frac{1}{2\left(\omega-\epsilon_{q}\right)}\left[\sum_{i j a} v_{i j}^{q a} x_{a}^{(1) i j}(\omega)_{p}+\frac{1}{\left(\omega-\epsilon_{p}\right)} \sum_{i a b} v_{a b}^{p i} t_{a b}^{(1) q i}\right] \\
x_{a}^{(1) i j}(\omega)_{p}=\frac{v_{p a}^{i j}}{\left(\omega-\epsilon_{p}\right)\left(\omega+\epsilon_{a}-\epsilon_{i}-\epsilon_{j}\right)} \\
t_{a b}^{(1) i j}=\lambda_{i j}^{(1) a b}=\frac{v_{a b}^{i j}}{\left(\epsilon_{i}+\epsilon_{j}-\epsilon_{a}-\epsilon_{b}\right)}=\frac{v_{a b}^{i j}}{\epsilon_{a b}^{i j}} \\
x_{a}^{(1) i j}(\omega)_{p}=\frac{v_{p a}^{i j}}{\left(\omega-\epsilon_{p}\right)\left(\omega+\epsilon_{a}-\epsilon_{i}-\epsilon_{j}\right)}
\end{gathered}
$$

The final Dyson and cumulant form of $G_{p q}^{R}(t)$ becomes pparent after inserting these expressions into Eq. (53) and proceeding as described in the main paper.

\section{E. One-particle simplification of the EOM-CCS equations}

Given the computational demand of the full EOM-CCS method, it is of interest to see if approximations other than those explored in the main paper are possible. In particular, approximations arising from effective one-body Hamiltonians since these are common in the works of Hedin, Nozieres, Langreth, etc. The full EOM-CCS method has the following matrix element for the computation of the amplitude variation:

$$
\begin{aligned}
\left\langle\phi_{i}^{a}\left|\bar{H}_{N}(t)\right| \phi\right\rangle= & f_{a i}+\sum_{b} f_{a b} t_{i}^{b}-\sum_{j} f_{j i} t_{j}^{a}-\sum_{j b} f_{j b} t_{i}^{b} t_{j}^{a} \\
& +\sum_{j b} v_{a j}^{i b} t_{j}^{b}-\sum_{j k b} v_{i b}^{j k} t_{j}^{a} t_{k}^{b}+\sum_{j b c} v_{a j}^{b c} t_{i}^{b} t_{j}^{c}-\sum_{j k b d} v_{j k}^{b d} t_{i}^{b} t_{j}^{a} t_{k}^{d},
\end{aligned}
$$

where the $f$ terms come from the one-particle part of $H$ and the $v$ terms from the two-

particle part. A simple, yet extreme approximation would be to only retain the first line in Eq. (59). However, this approximation leaves out the important linear term in $v$. We now partition that term as follows:

$$
\sum_{j b} v_{a j}^{i b} t_{j}^{b}=v_{i a}^{i a} t_{i}^{a}+\sum_{j} v_{a j}^{i a} t_{j}^{a}+\sum_{b} v_{a i}^{i b} t_{i}^{b}+\sum_{j b(\neq i a)} v_{a j}^{i b} t_{j}^{b}
$$

Discarding the last term and introducing the approximate sum into the matrix element expression above while only keeping the $f$ terms we obtain:

$$
\left\langle\phi_{i}^{a}\left|\bar{H}_{N}(t)\right| \phi\right\rangle=f_{a i}+v_{i a}^{i a} t_{i}^{a}+\sum_{b}\left(f_{a b}-v_{a j}^{b i}\right) t_{i}^{b}-\sum_{j}\left(f_{j i}-v_{a j}^{i a}\right) t_{j}^{a}-\sum_{j b} f_{j b} t_{i}^{b} t_{j}^{a}
$$




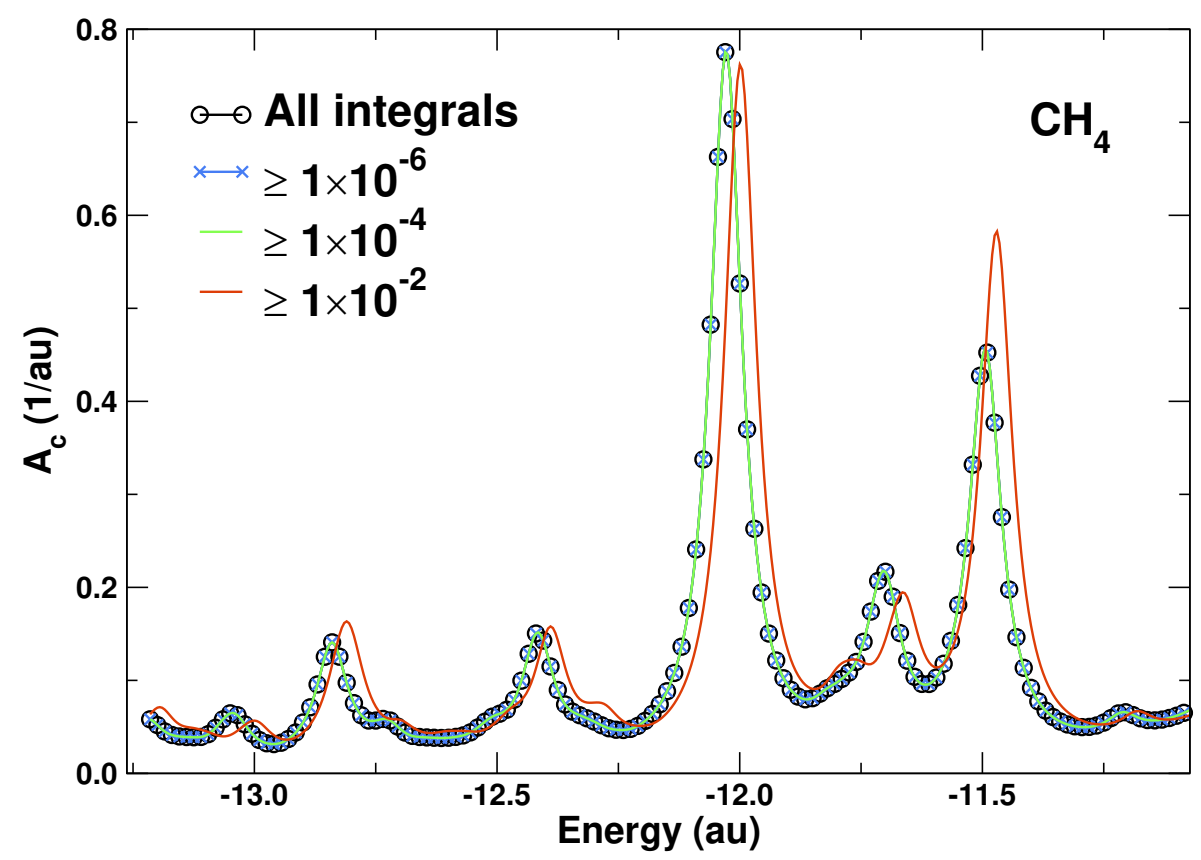

FIG. 3: Comparison of the satellite region of the core spectral function of $\mathrm{CH}_{4}$ computed with the cc-pVDZ basis set and the full EOM-CCS method, as a function of potential integral cutoff.

Therefore, with this approximation the form of the one-particle $H$ is preserved, but with modified $f$ elements, which now include a correction for the particular $(i, a)$ valence-valence excitation. We are currently exploring the possibility of using this simplified propagation form with $f$ and $v$ parameters from effective Hamiltonians.

\section{RESULTS}

\section{A. Effect of potential integral trimming}

As discussed in the main manuscript, in order to reduce the storage requirements and computational demands of the EOM-CC method, we screen those $v_{p q}^{r s}$ integrals below a certain threshold after the SCF is fully converged. Figure 3 shows a comparison between the spectral function of a typical system $\left(\mathrm{CH}_{4}\right)$ for different values of the cutoff parameter. We find that for the cutoff used in this paper $\left(1 \times 10^{-4} \mathrm{au}\right)$ the EOM-CC results are indistinguishable from those obtained with all the integrals. With this cutoff, however, the performance of the method is increase by a factor of 10. This cutoff is also used in the DSE2 calculations, 
TABLE I: Comparison of the experimental core binding energies (in $\mathrm{eV}$ ) to those obtained with the DZVP basis set, using the L and NL approximations to the cumulant and the 1-3 approximations of the EOM-CCS method, and their mean absolute errors (MAE).

\begin{tabular}{|c|c|c|c|c|c|c|c|c|c|c|}
\hline System & $\mathbf{K T}$ & DSE2 & $\mathbf{1}_{L}$ & $\mathbf{2}_{L}$ & $\mathbf{3}_{L}$ & $\mathbf{1}_{N L}$ & $\mathbf{2}_{N L}$ & $\mathbf{3}_{N L}$ & Expt & Ref \\
\hline $\mathrm{CH}_{4}$ & 304.744 & 291.881 & 286.990 & 287.425 & 286.994 & 290.412 & 290.679 & 290.415 & 290.703 & {$[5]$} \\
\hline $\mathrm{NH}_{3}$ & 422.523 & 405.466 & 400.603 & 400.815 & 400.198 & 405.057 & 405.177 & 404.816 & 405.520 & {$[6]$} \\
\hline $\mathrm{H}_{2} \mathrm{O}$ & 559.003 & 538.597 & 534.795 & 534.390 & 533.705 & 539.498 & 539.248 & 538.843 & 539.700 & {$[7]$} \\
\hline $\mathrm{HF}$ & 714.753 & 692.127 & 689.876 & 688.904 & 688.313 & 694.174 & 693.549 & 693.178 & 694.200 & {$[8]$} \\
\hline $\mathrm{Ne}$ & 890.987 & 868.010 & 867.661 & 866.444 & 866.109 & 870.935 & 870.076 & 869.842 & 870.200 & {$[9]$} \\
\hline MAE & 18.34 & 1.32 & 4.08 & 4.47 & 5.00 & 0.34 & 0.32 & 0.65 & & \\
\hline
\end{tabular}

where it also has little effect in the accuracy, but produced only a modest improvement in performance. It should be noted that this integral trimming is not used in the GFCCSD and GFCC-i $(2,3)$ calculations.

\section{B. Quasiparticle properties with the DZVP and cc-pVDZ basis sets}

Tables I and II summarize the core binding energies for the different systems and methods calculated with the DZVP and cc-pVDZ basis sets, respectively. While the KT, DSE2, and EOM-CC results show little dependence on the basis set, for the GFCC methods the augmented Dunning basis set seems not able to improve the results. To reduce the discrepancy of the GFCC results, the employment of bare Dunning basis sets seems slightly better. For the GFCCSD results, employing the aug-cc-pVDZ basis gives the MAE of 4.24 $\mathrm{eV}$, in comparison to the MAE of $3.57 \mathrm{eV}$ brought by employing the bare cc-pVDZ basis. Furthermore, the triple- $\zeta$ cc-pVTZ basis can systematically reduce the discrepancies to even below $1 \mathrm{eV}(\sim 0.74 \mathrm{eV})$, which agrees with basis set discussion in the previous EOM-CC and GFCC results for the core ionizations of small molecules. ${ }^{2-4}$

Tables III and IV summarize the core quasiparticle strengths for the different systems and methods calculated with the DZVP and cc-pVDZ basis sets, respectively. 
TABLE II: Comparison of the experimental core binding energies (in eV) to those obtained with the cc-pVDZ basis set, using the $\mathrm{L}$ and NL approximations to the cumulant and the $\mathrm{L}_{c}, \mathrm{Q}$ and $\mathrm{F}$ approximations of the EOM-CCS method, and their mean absolute errors (MAE). The KT results are obtained with Koopmans' Theorem.

\begin{tabular}{|c|c|c|c|c|c|c|c|c|c|c|c|}
\hline System & $\mathbf{K T}$ & DSE2 & GFCCSD & $\operatorname{GFCC}-\mathrm{i}(2,3)$ & $\mathbf{1}_{N L}$ & $\mathbf{2}_{L}$ & $\mathbf{3}_{L}$ & $\mathbf{1}_{L}$ & $\mathbf{2}_{N L}$ & $\mathbf{3}_{N L}$ & Expt \\
\hline $\mathrm{CH}_{4}$ & 305.17 & 292.56 & 292.80 & 293.45 & 286.98 & 287.84 & 287.44 & 290.54 & 291.08 & 290.83 & 290.703 \\
\hline $\mathrm{NH}_{3}$ & 422.78 & 406.26 & 407.68 & 408.61 & 400.67 & 401.35 & 400.81 & 405.13 & 405.55 & 405.23 & 405.520 \\
\hline $\mathrm{H}_{2} \mathrm{O}$ & 559.25 & 539.30 & 542.21 & 543.41 & 534.53 & 534.74 & 534.15 & 539.32 & 539.46 & 539.10 & 539.700 \\
\hline $\mathrm{HF}$ & 715.09 & 692.64 & 696.78 & 698.19 & 689.27 & 688.97 & 688.45 & 693.78 & 693.59 & 693.27 & 694.200 \\
\hline $\mathrm{Ne}$ & 891.59 & 868.17 & 874.52 & 874.52 & 866.50 & 865.87 & 865.57 & 870.16 & 869.73 & 869.52 & 870.200 \\
\hline MAE & 18.71 & 1.32 & 2.73 & 3.57 & 4.48 & 4.31 & 4.78 & 0.28 & 0.35 & 0.53 & \\
\hline
\end{tabular}

TABLE III: Comparison of the quasiparticle strengths obtained with the DZVP basis set, using the L and NL approximations to the cumulant and the 1-3 approximations of the EOM-CCS method.

\begin{tabular}{lccccccc}
\hline \hline System & DSE2 & $\mathbf{1}_{L}$ & $\mathbf{2}_{L}$ & $\mathbf{3}_{L}$ & $\mathbf{1}_{N L}$ & $\mathbf{2}_{N L}$ & $\mathbf{3}_{N L}$ \\
\hline $\mathrm{CH}_{4}$ & 0.79 & 0.60 & 0.61 & 0.59 & 0.70 & 0.71 & 0.69 \\
$\mathrm{NH}_{3}$ & 0.77 & 0.60 & 0.61 & 0.58 & 0.71 & 0.71 & 0.69 \\
$\mathrm{H}_{2} \mathrm{O}$ & 0.75 & 0.63 & 0.62 & 0.59 & 0.73 & 0.72 & 0.70 \\
$\mathrm{HF}$ & 0.76 & 0.68 & 0.66 & 0.64 & 0.76 & 0.74 & 0.72 \\
$\mathrm{Ne}$ & 0.78 & 0.76 & 0.73 & 0.72 & 0.80 & 0.78 & 0.77 \\
\hline \hline
\end{tabular}

C. Full comparison of the spectral function as a function of basis set, level of CCS approximation and cumulant form

Figures 4 and 5 shows a comparison of the core spectral function of the $10 e$ systems computed with the DZVP and cc-pVDZ basis sets, respectively, and the $3_{N L}$ approach, as a function of EOM-CCS approximation. 
TABLE IV: Comparison of the quasiparticle strengths obtained with the cc-pVDZ basis set, using the L and NL approximations to the cumulant and the 1-3 approximations of the EOM-CCS method.

\begin{tabular}{lccccccccc}
\hline \hline System & DSE2 & GFCCSD & GFCC-i(2,3) & $\mathbf{1}_{L}$ & $\mathbf{2}_{L}$ & $\mathbf{3}_{L}$ & $\mathbf{1}_{N L}$ & $\mathbf{2}_{N L}$ & $\mathbf{3}_{N L}$ \\
\hline $\mathrm{CH}_{4}$ & 0.80 & 0.77 & 0.81 & 0.60 & 0.63 & 0.61 & 0.70 & 0.72 & 0.71 \\
$\mathrm{NH}_{3}$ & 0.78 & 0.76 & 0.81 & 0.61 & 0.63 & 0.61 & 0.71 & 0.73 & 0.71 \\
$\mathrm{H}_{2} \mathrm{O}$ & 0.78 & 0.71 & 0.82 & 0.64 & 0.65 & 0.63 & 0.74 & 0.74 & 0.72 \\
$\mathrm{HF}$ & 0.79 & 0.79 & 0.84 & 0.70 & 0.69 & 0.67 & 0.77 & 0.76 & 0.75 \\
$\mathrm{Ne}$ & 0.81 & 1.00 & 1.00 & 0.77 & 0.76 & 0.75 & 0.82 & 0.81 & 0.80 \\
\hline \hline
\end{tabular}

TABLE V: Scissors corrections used in Fig. 6.

\begin{tabular}{lcc}
\hline \hline System & $\mathbf{1}_{N L}$ & $\mathbf{2}_{N L}$ \\
\hline $\mathrm{CH}_{4}$ & 1.9 & 1.1 \\
$\mathrm{NH}_{3}$ & 6.2 & 1.6 \\
$\mathrm{H}_{2} \mathrm{O}$ & 8.8 & 1.8 \\
$\mathrm{HF}$ & 10.4 & 2.1 \\
$\mathrm{Ne}$ & 10.2 & 1.5 \\
\hline \hline
\end{tabular}

D. Effect of the CCS approach on the gap between the quasiparticle and the satellites

Figure 6 shows a comparison of the spectral functions with a scissors correction applied to the $1_{N L}$ and $2_{N L}$ approaches in such a way that the satellite regions become aligned with those in the $3_{N L}$ approach. After the correction is applied, the $2_{N L}$ approximation is shown to give results that are almost identical to the full approach. Despite showing some noticeable differences, $1_{N L}$ approximation shows reasonable agreement in the overall distribution of the satellite intensity. The scissors corrections for each system and method are shown in Table V. Interestingly, while the corrections required for the $1_{N L}$ approximation are clearly system-dependent, in the case of the $2_{N L}$ approximation the corrections are almost constant, suggesting that an overall scissors correction can be used to simulate the results of the more expensive full approach. 


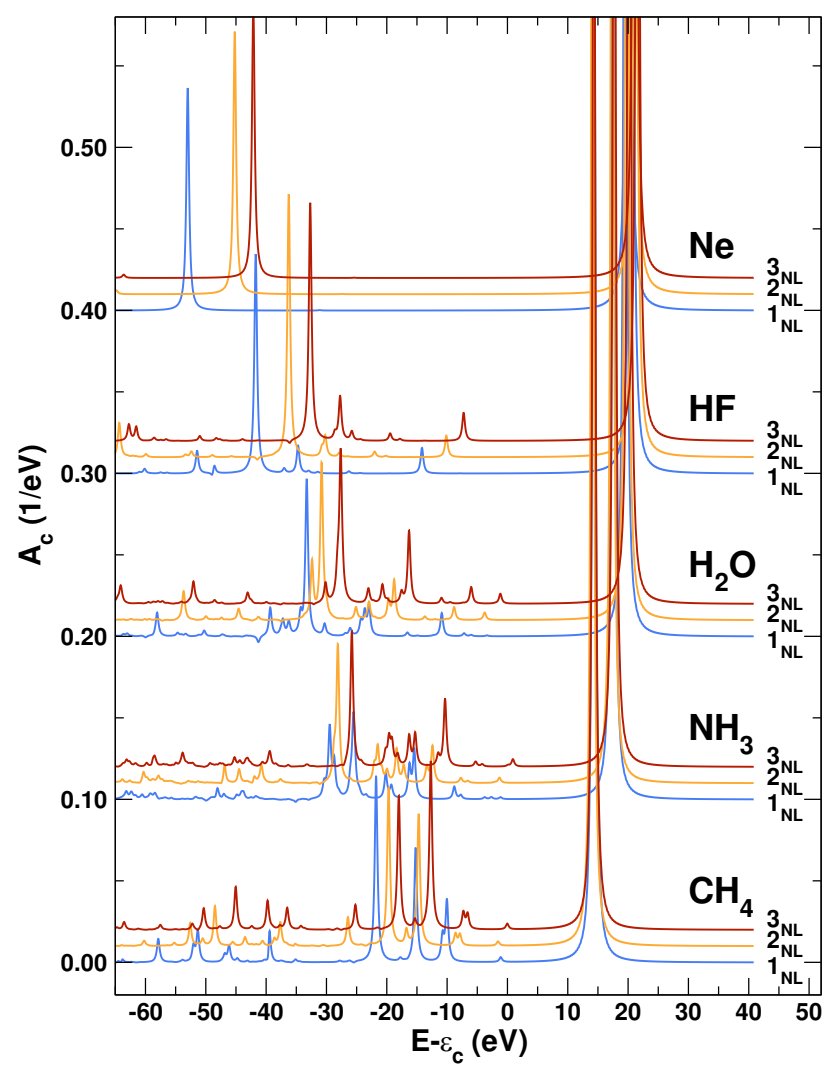

FIG. 4: Core spectral function $A_{c}$ of the $10 e$ systems computed with the DZVP basis set, as a function of EOM-CCS approximation $1_{N L}$ (blue), $2_{N L}$ (orange), and $3_{N L}$ (red).

1 B. Peng and K. Kowalski, Phys. Rev. A 94, 062512 (2016).

2 S. Sen, A. Shee, and D. Mukherjee, Mol. Phys. 111, 2625 (2013).

3 S. Coriani and H. Koch, J. Chem. Phys. 145, 149901 (2016).

4 B. Peng and K. Kowalski, J. Chem. Theory Comput. 14, 4335 (2018), pMID: 29957945.

5 T. Karlsen, K. J. Brve, L. J. Sthre, K. Wiesner, M. Bssler, and S. Svensson, Journal of the American Chemical Society 124, 7866 (2002).

6 T. Buttersack, P. E. Mason, R. S. McMullen, T. Martinek, K. Brezina, D. Hein, H. Ali, C. Kolbeck, C. Schewe, S. Malerz, et al., Journal of the American Chemical Society 141, 1838 (2019).

7 F. Vies, C. Sousa, and F. Illas, Phys. Chem. Chem. Phys. 20, 8403 (2018).

8 Atomic Data and Nuclear Data Tables 31, 433 (1984), ISSN 0092-640X.

9 G. P. Williams, X-Ray Data Booklet: Section 1.1 ELECTRON BINDING ENERGIES (Lawrence 


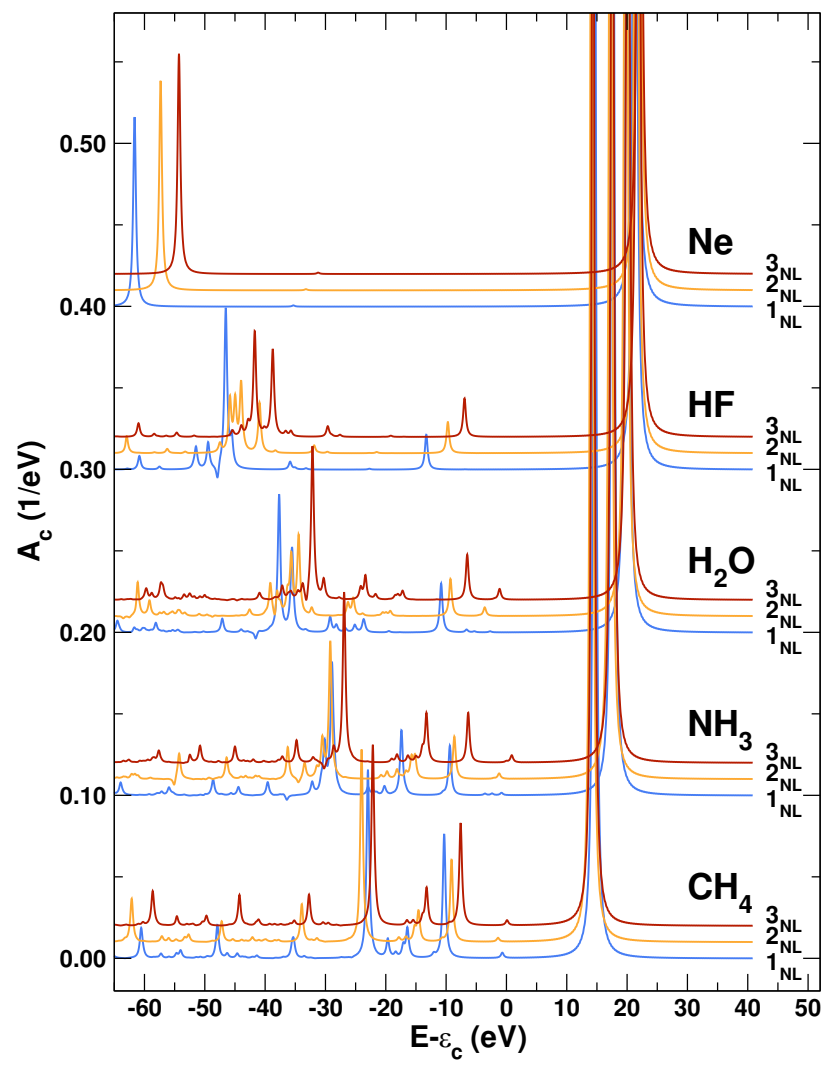

FIG. 5: Core spectral function $A_{c}$ of the $10 e$ systems computed with the cc-pVDZ basis set, as a function of EOM-CCS approximation $1_{N L}$ (blue), $2_{N L}$ (orange), and $3_{N L}$ (red).

Berkeley National Laboratory, Berkeley, 2009). 


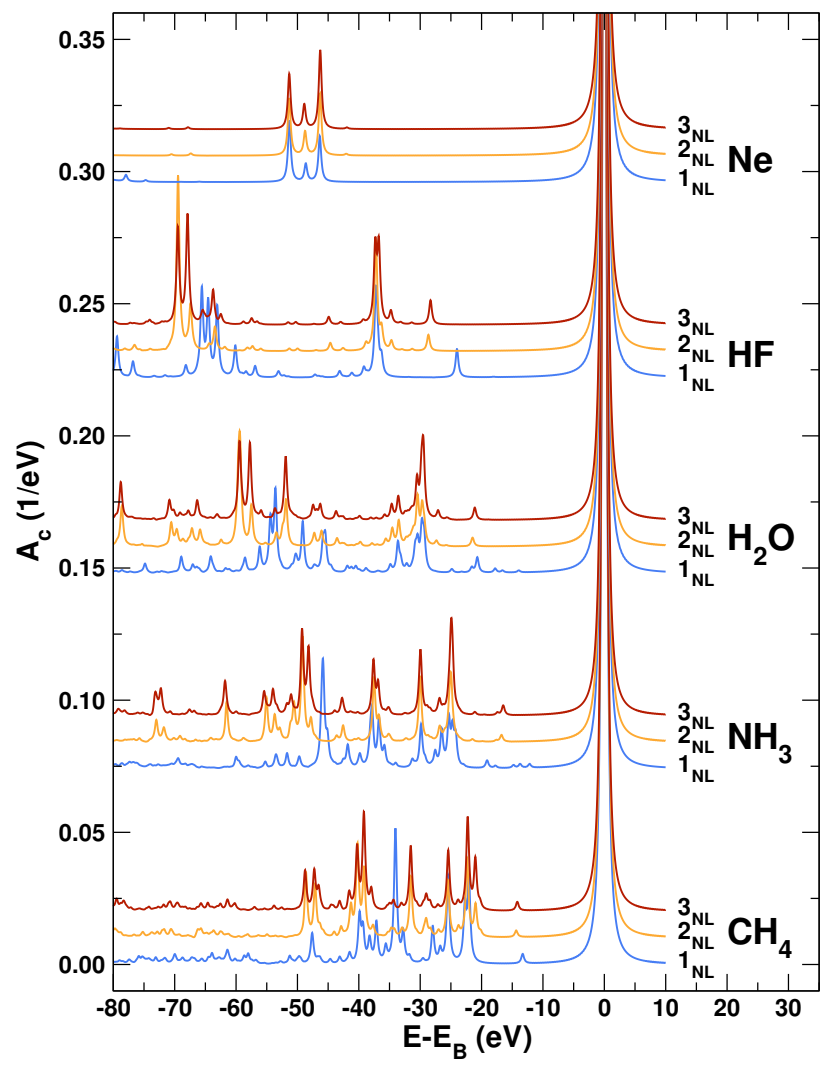

FIG. 6: Comparison of the spectral functions with a scissors correction (see Table V) applied to the $1_{N L}$ (blue) and $2_{N L}$ (orange) approximations in such a way that the satellite regions become aligned with those of $3_{N L}$ (red). 